\title{
A stochastic Galerkin method for the Boltzmann equation with uncertainty*
}

\author{
Jingwei $\mathrm{Hu}^{\dagger}$ and Shi Jin ${ }^{\ddagger}$
}

March 20, 2016

\begin{abstract}
We develop a stochastic Galerkin method for the Boltzmann equation with uncertainty. The method is based on the generalized polynomial chaos (gPC) approximation in the stochastic Galerkin framework, and can handle random inputs from collision kernel, initial data or boundary data. We show that a simple singular value decomposition of gPC related coefficients combined with the fast Fourier-spectral method (in velocity space) allows one to compute the high-dimensional collision operator very efficiently. In the spatially homogeneous case, we first prove that the analytical solution preserves the regularity of the initial data in the random space, and then use it to establish the spectral accuracy of the proposed stochastic Galerkin method. Several numerical examples are presented to illustrate the validity of the proposed scheme.
\end{abstract}

Key words. Uncertainty quantification, the Boltzmann equation, random input, generalized polynomial chaos, stochastic Galerkin method, singular value decomposition, fast spectral method.

AMS subject classifications. 35Q20, 65M70.

\section{Introduction}

Kinetic theory is an indispensable tool to describe the non-equilibrium dynamics of a gas or system comprised of a large number of particles, primarily through applications of the Boltzmann equation [4]. When the well-known fluid mechanics laws of Navier-Stokes and Fourier become inadequate to represent the system, this equation can provide reliable information at the mesoscopic level, and is widely used in the fields such as rarefied gas dynamics, astronautical engineering, etc.

While research studies on mathematical theory $[7,6,31]$ and numerical approximation $[5,9]$ of the Boltzmann equation are extensive and still ongoing, they almost exclusively concern the deterministic problem. To authors' best knowledge, no research has been conducted to evaluate

\footnotetext{
${ }^{*}$ This work was partially supported by the NSF grant RNMS-1107291 (KI-Net).

$\dagger$ Department of Mathematics, Purdue University, West Lafayette, IN 47907, USA (jingweihu@purdue.edu). This author's research was also supported by a startup grant from Purdue University.

$\ddagger$ Department of Mathematics, University of Wisconsin-Madison, Madison, WI 53706, USA; Department of Mathematics, Institute of Natural Sciences, MOE-LSEC and SHL-MAC, Shanghai Jiao Tong University, Shanghai 200240, China (sjin@wisc.edu). This author's research was also supported by NSF grant 1114546 and NSFC grant 91330203.

(C) 2016. This manuscript version is made available under the Elsevier user license http://www.elsevier.com/open-access/userlicense/1.0/
} 
the impact of random inputs to the equation. There are, however, many sources that can bring this kind of uncertainties. For example, one of the key terms in the Boltzmann equation is the collision kernel (or cross section) which characterizes the law of interaction between particles. Ideally, it should be computed from the intermolecular potential using the scattering theory [18]. In practice, for simplicity, phenomenological collision kernels are often used with the aim to reproduce viscosity and diffusion coefficients $[2,22]$. Specifically, these kernels contain adjustable parameters whose values can be determined by matching with the available transport data for various gases. On the other hand, when considering time evolution of a gas in bounded domains, one needs to supplement with appropriate initial and boundary conditions. These are usually given in terms of measured values of macroscopic quantities such as density, temperature, bulk velocity, etc (e.g., shock tube and Couette flow problems). In either case, one has to rely on the experimental data which inevitably involve uncertainties due to measurement errors. Therefore, it is interesting to study the behavior of the solution to the Boltzmann equation when randomness presents in the collision kernel or initial/boundary data. Most importantly, a proper quantification of these uncertainties will allow scientists and engineers to obtain more reliable predictions for sensitivity analysis and risk managements.

This paper serves as a first attempt to conduct uncertainty quantification (UQ) for the Boltzmann equation with random inputs. We will adopt the generalized polynomial chaos (gPC) based stochastic Galerkin approximation, which has been successfully applied to many physical and engineering problems, see for instance, the overviews in [16,33]. We also mention that the same technique was used recently to solve some linear kinetic models in the diffusive limit [21]. In the spatially homogeneous case, we will first prove that the analytical solution preserves the regularity of the initial data in the random space, and then use it to establish the spectral accuracy of the proposed stochastic Galerkin method.

For the Boltzmann equation, the main difficulty lies in its high-dimensional nonlinear collision integral, whose direct evaluation would be prohibitively expensive. We will show that a simple singular value decomposition (SVD) of gPC related coefficients combined with the fast spectral method in the deterministic case [28] enables one to compute the collision operator accurately and efficiently. Specifically, we are able to reduce the computational cost from $O\left(N_{K}^{2} N_{\sigma}^{d-1} N_{\mathbf{v}}^{2 d}\right)$ to $\max \left\{O\left(R_{\mathbf{k}} N_{\sigma}^{d-1} N_{\mathbf{v}}^{d} \log N_{\mathbf{v}}\right), O\left(R_{\mathbf{k}} N_{K} N_{\mathbf{v}}^{d}\right)\right\}$, for some $R_{\mathbf{k}} \leq N_{K}$, where $N_{K}=\left(\begin{array}{c}K+n \\ K\end{array}\right)$ is the dimension of $n$-variate polynomials of degree up to $K, d$ is the dimension of velocity space (typically $d=2$ or 3 ), $N_{\sigma}$ is the number of discrete points in each angular direction, and $N_{\mathbf{v}}$ is the number of points in each velocity dimension (typically $N_{\sigma} \ll N_{\mathbf{v}}$ ).

The rest of this paper is organized as follows. In the next section, we introduce the Boltzmann equation with random inputs and formulate the UQ problem systematically. Section 3 describes in detail the gPC based stochastic Galerkin scheme, where the fast algorithm for the collision operator as well as the time/spatial discretization are discussed. In section 4 , we first study the regularity in the random space of the analytical solution and then use it to establish the spectral convergence of the stochastic Galerkin method. Extensive numerical examples are presented in section 5 to illustrate the validity of the proposed scheme. Finally some conclusions are given in section 6 . 


\section{The Boltzmann equation with random inputs}

Our starting point is the following (dimensionless) deterministic Boltzmann equation [4, 31], which is the central model in kinetic theory and describes the time evolution of a rarefied gas:

$$
\frac{\partial f}{\partial t}+\mathbf{v} \cdot \nabla_{\mathbf{x}} f=\frac{1}{\mathrm{Kn}} \mathcal{Q}(f, f), \quad t>0, \mathbf{x}, \mathbf{v} \in \mathbb{R}^{d}, d=2 \text { or } 3,
$$

where $f=f(t, \mathbf{x}, \mathbf{v})$ is the phase space distribution function of particles at time $t$, position $\mathbf{x}$, and velocity $\mathbf{v} . \mathrm{Kn}$ is the Knudsen number defined as the ratio of mean free path and typical length scale of the problem. $\mathcal{Q}(f, f)$ is the nonlinear (quadratic) Boltzmann collision operator modeling the binary interactions between particles (since $\mathcal{Q}$ acts only in the velocity space, variables $t$ and $\mathbf{x}$ are suppressed from the functions below):

$$
\mathcal{Q}(f, g)(\mathbf{v})=\int_{\mathbb{R}^{d}} \int_{S^{d-1}} B\left(\mathbf{v}-\mathbf{v}_{*}, \sigma\right)\left[f\left(\mathbf{v}^{\prime}\right) g\left(\mathbf{v}_{*}^{\prime}\right)-f(\mathbf{v}) g\left(\mathbf{v}_{*}\right)\right] \mathrm{d} \sigma \mathrm{d} \mathbf{v}_{*} .
$$

Here $\left(\mathbf{v}, \mathbf{v}_{*}\right)$ and $\left(\mathbf{v}^{\prime}, \mathbf{v}_{*}^{\prime}\right)$ are the velocity pairs before and after collision, during which the momentum and energy are conserved; hence $\left(\mathbf{v}^{\prime}, \mathbf{v}_{*}^{\prime}\right)$ can be represented in terms of $\left(\mathbf{v}, \mathbf{v}_{*}\right)$ as

$$
\left\{\begin{array}{l}
\mathbf{v}^{\prime}=\frac{\mathbf{v}+\mathbf{v}_{*}}{2}+\frac{\left|\mathbf{v}-\mathbf{v}_{*}\right|}{2} \sigma \\
\mathbf{v}_{*}^{\prime}=\frac{\mathbf{v}+\mathbf{v}_{*}}{2}-\frac{\left|\mathbf{v}-\mathbf{v}_{*}\right|}{2} \sigma
\end{array}\right.
$$

with the parameter $\sigma$ varying in the unit sphere $S^{d-1}$. The collision kernel $B\left(\mathbf{v}-\mathbf{v}_{*}, \sigma\right)$ is a non-negative function depending only on $\left|\mathbf{v}-\mathbf{v}_{*}\right|$ and cosine of the deviation angle $\theta$ :

$$
B\left(\mathbf{v}-\mathbf{v}_{*}, \sigma\right)=B\left(\left|\mathbf{v}-\mathbf{v}_{*}\right|, \cos \theta\right), \quad \cos \theta=\frac{\sigma \cdot\left(\mathbf{v}-\mathbf{v}_{*}\right)}{\left|\mathbf{v}-\mathbf{v}_{*}\right|} .
$$

The specific form of $B$ is determined from the intermolecular potential via the scattering theory. For numerical purpose, a commonly used model is the variable hard-sphere (VHS) model introduced by Bird [2]:

$$
B\left(\left|\mathbf{v}-\mathbf{v}_{*}\right|, \cos \theta\right)=b_{\lambda}\left|\mathbf{v}-\mathbf{v}_{*}\right|^{\lambda}, \quad-d<\lambda \leq 1,
$$

where $b_{\lambda}$ is a constant, $\lambda>0$ corresponds to the hard potentials, and $\lambda<0$ to the soft potentials.

The collision operator (2.2) conserves mass, momentum, and energy:

$$
\int_{\mathbb{R}^{d}} \mathcal{Q}(f, f) \mathrm{d} \mathbf{v}=\int_{\mathbb{R}^{d}} \mathcal{Q}(f, f) \mathbf{v} \mathrm{d} \mathbf{v}=\int_{\mathbb{R}^{d}} \mathcal{Q}(f, f)|\mathbf{v}|^{2} \mathrm{~d} \mathbf{v}=0 .
$$

Moreover, it satisfies the celebrated Boltzmann's $H$-theorem:

$$
-\int_{\mathbb{R}^{d}} \mathcal{Q}(f, f) \ln f \mathrm{~d} \mathbf{v} \geq 0
$$

which implies that the entropy is always increasing, and reaches its maximum if and only if $f$ attains the local equilibrium:

$$
\mathcal{M}(\mathbf{v})_{(\rho, \mathbf{u}, T)}=\frac{\rho}{(2 \pi T)^{d / 2}} e^{-\frac{(\mathbf{v}-\mathbf{u})^{2}}{2 T}}
$$


where $\rho, \mathbf{u}, T$ are, respectively, the density, bulk velocity, and temperature defined by

$$
\rho=\int_{\mathbb{R}^{d}} f(\mathbf{v}) \mathrm{d} \mathbf{v}, \quad \mathbf{u}=\frac{1}{\rho} \int_{\mathbb{R}^{d}} f(\mathbf{v}) \mathbf{v} \mathrm{d} \mathbf{v}, \quad T=\frac{1}{d \rho} \int_{\mathbb{R}^{d}} f(\mathbf{v})|\mathbf{v}-\mathbf{u}|^{2} \mathrm{~d} \mathbf{v} .
$$

When a Cauchy problem is considered, the equation (2.1) needs to be supplemented with an initial condition:

$$
f(0, \mathbf{x}, \mathbf{v})=f^{0}(\mathbf{x}, \mathbf{v}) .
$$

If we further consider a bounded spatial domain $\mathbf{x} \in \Omega \subset \mathbb{R}^{d}$, then a proper boundary condition also needs to be imposed. A widely used one for the Boltzmann equation is the so-called Maxwell boundary condition: for any boundary point $\mathbf{x} \in \partial \Omega$, let $n(\mathbf{x})$ be the unit normal vector to the boundary, pointed to the gas, then the in-flow boundary condition is specified as

$$
f(t, \mathbf{x}, \mathbf{v})=g(t, \mathbf{x}, \mathbf{v}), \quad\left(\mathbf{v}-\mathbf{u}_{w}\right) \cdot n>0,
$$

and

$$
\begin{aligned}
g(t, \mathbf{x}, \mathbf{v}):= & (1-\alpha) f\left(t, \mathbf{x}, \mathbf{v}-2\left[\left(\mathbf{v}-\mathbf{u}_{w}\right) \cdot n\right] n\right) \\
& +\frac{\alpha}{(2 \pi)^{\frac{d-1}{2}} T_{w}^{\frac{d+1}{2}}} e^{-\frac{\left|\mathbf{v}-\mathbf{u}_{w}\right|^{2}}{2 T_{w}}} \int_{\left(\mathbf{v}-\mathbf{u}_{w}\right) \cdot n<0} f(t, \mathbf{x}, \mathbf{v})\left|\left(\mathbf{v}-\mathbf{u}_{w}\right) \cdot n\right| \mathrm{d} \mathbf{v},
\end{aligned}
$$

where $\mathbf{u}_{w}=\mathbf{u}_{w}(t, \mathbf{x}), T_{w}=T_{w}(t, \mathbf{x})$ are, respectively, the velocity and temperature of the wall (boundary). The constant $\alpha(0 \leq \alpha \leq 1)$ is the accommodation coefficient with $\alpha=$ 1 corresponding to the purely diffusive boundary, and $\alpha=0$ the purely specular reflective boundary.

Later we will also need the asymmetric collision operator:

$$
\mathcal{Q}(g, h)(\mathbf{v})=\int_{\mathbb{R}^{d}} \int_{S^{d-1}} B\left(\mathbf{v}-\mathbf{v}_{*}, \sigma\right)\left[g\left(\mathbf{v}^{\prime}\right) h\left(\mathbf{v}_{*}^{\prime}\right)-g(\mathbf{v}) h\left(\mathbf{v}_{*}\right)\right] \mathrm{d} \sigma \mathrm{d} \mathbf{v}_{*} .
$$

As mentioned in the introduction, for real-world problems, the collision kernel, initial data, or boundary data may contain uncertainties that propagate into the solution and affect its property substantially. To quantify these uncertainties, we formulate our problem in a stochastic manner [33] as follows:

$$
\left\{\begin{array}{l}
\frac{\partial f}{\partial t}+\mathbf{v} \cdot \nabla_{\mathbf{x}} f=\frac{1}{\mathrm{Kn}} \mathcal{Q}(f, f)(t, \mathbf{x}, \mathbf{v}, \mathbf{z}), \quad t>0, \mathbf{x} \in \Omega, \mathbf{v} \in \mathbb{R}^{d}, \mathbf{z} \in I_{\mathbf{z}}, \\
f(0, \mathbf{x}, \mathbf{v}, \mathbf{z})=f^{0}(\mathbf{x}, \mathbf{v}, \mathbf{z}), \quad \mathbf{x} \in \Omega, \mathbf{v} \in \mathbb{R}^{d}, \mathbf{z} \in I_{\mathbf{z}}, \\
f(t, \mathbf{x}, \mathbf{v}, \mathbf{z})=g(t, \mathbf{x}, \mathbf{v}, \mathbf{z}), \quad t \geq 0, \mathbf{x} \in \partial \Omega, \mathbf{v} \in \mathbb{R}^{d}, \mathbf{z} \in I_{\mathbf{z}} .
\end{array}\right.
$$

Now $f=f(t, \mathbf{x}, \mathbf{v}, \mathbf{z})$ depends on an extra variable $\mathbf{z}$ - an $n$-dimensional random vector with support $I_{\mathbf{z}}$ characterizing the random inputs of the system. The randomness could come from

- the collision kernel, for instance, $B=b_{\lambda}\left(\mathbf{z}^{B}\right)\left|\mathbf{v}-\mathbf{v}_{*}\right|^{\lambda}$;

- the boundary data $g(t, \mathbf{x}, \mathbf{v}, \mathbf{z})$, in such a way that $\mathbf{u}_{w}, T_{w}$ in (2.5) are replaced by $\mathbf{u}_{w}\left(t, \mathbf{x}, \mathbf{z}^{b}\right)$ and $T_{w}\left(t, \mathbf{x}, \mathbf{z}^{b}\right)$;

- the initial data $f^{0}(\mathbf{x}, \mathbf{v}, \mathbf{z})$, via initial macroscopic quantities: density $\rho^{0}\left(\mathbf{x}, \mathbf{z}^{i}\right)$, temperature $T^{0}\left(\mathbf{x}, \mathbf{z}^{i}\right)$, etc. 
Therefore, $\mathbf{z}$ is a collection of random vectors $\mathbf{z}^{B}, \mathbf{z}^{b}$ and $\mathbf{z}^{i}$, summed up to dimension $n$, i.e., $\mathbf{z}:=\left(\mathbf{z}^{B}, \mathbf{z}^{b}, \mathbf{z}^{i}\right)=\left(z_{1}, \ldots, z_{n}\right)$. For simplicity, we assume in this paper, the components of $\mathbf{z}$ are mutually independent random variables already obtained through some dimension reduction

technique, e.g., Karhunen-Loève expansion [24], and do not pursue further the issue of random input parameterization.

\section{A gPC based stochastic Galerkin method}

In this section, we construct a stochastic Galerkin scheme for the problem (2.7) using gPC expansion. Specifically, we seek a solution in the following form:

$$
f(t, \mathbf{x}, \mathbf{v}, \mathbf{z}) \approx \sum_{|\mathbf{k}|=0}^{K} f_{\mathbf{k}}(t, \mathbf{x}, \mathbf{v}) \Phi_{\mathbf{k}}(\mathbf{z}):=f^{K}(t, \mathbf{x}, \mathbf{v}, \mathbf{z}) .
$$

Here $\mathbf{k}=\left(k_{1}, \ldots, k_{n}\right)$ is a multi-index with $|\mathbf{k}|=k_{1}+\cdots+k_{n} .\left\{\Phi_{\mathbf{k}}(\mathbf{z})\right\}$ are orthonormal gPC basis functions satisfying

$$
\int_{I_{\mathbf{z}}} \Phi_{\mathbf{k}}(\mathbf{z}) \Phi_{\mathbf{j}}(\mathbf{z}) \pi(\mathbf{z}) \mathrm{d} \mathbf{z}=\delta_{\mathbf{k j}}, \quad 0 \leq|\mathbf{k}|,|\mathbf{j}| \leq K,
$$

where $\pi(\mathbf{z})$ is the probability distribution function of $\mathbf{z}$. Inserting (3.1) into (2.7), and performing a standard Galerkin projection, we obtain

$$
\left\{\begin{array}{l}
\frac{\partial f_{\mathbf{k}}}{\partial t}+\mathbf{v} \cdot \nabla_{\mathbf{x}} f_{\mathbf{k}}=\frac{1}{\mathrm{Kn}} Q_{\mathbf{k}}\left(f^{K}, f^{K}\right)(t, \mathbf{x}, \mathbf{v}), \quad t>0, \mathbf{x} \in \Omega, \mathbf{v} \in \mathbb{R}^{d} \\
f_{\mathbf{k}}(0, \mathbf{x}, \mathbf{v})=f_{\mathbf{k}}^{0}(\mathbf{x}, \mathbf{v}), \quad \mathbf{x} \in \Omega, \mathbf{v} \in \mathbb{R}^{d}, \\
f_{\mathbf{k}}(t, \mathbf{x}, \mathbf{v})=g_{\mathbf{k}}(t, \mathbf{x}, \mathbf{v}), \quad t \geq 0, \mathbf{x} \in \partial \Omega, \mathbf{v} \in \mathbb{R}^{d}
\end{array}\right.
$$

for each $0 \leq|\mathbf{k}| \leq K$, and

$$
\begin{aligned}
& Q_{\mathbf{k}}\left(f^{K}, f^{K}\right):=\int_{I_{\mathbf{z}}} \mathcal{Q}\left(f^{K}, f^{K}\right)(t, \mathbf{x}, \mathbf{v}, \mathbf{z}) \Phi_{\mathbf{k}}(\mathbf{z}) \pi(\mathbf{z}) \mathrm{d} \mathbf{z} \\
& f_{\mathbf{k}}^{0}:=\int_{I_{\mathbf{z}}} f^{0}(\mathbf{x}, \mathbf{v}, \mathbf{z}) \Phi_{\mathbf{k}}(\mathbf{z}) \pi(\mathbf{z}) \mathrm{d} \mathbf{z} \\
& g_{\mathbf{k}}:=\int_{I_{\mathbf{z}}} g(t, \mathbf{x}, \mathbf{v}, \mathbf{z}) \Phi_{\mathbf{k}}(\mathbf{z}) \pi(\mathbf{z}) \mathrm{d} \mathbf{z}
\end{aligned}
$$

Remark 3.1. $Q_{\mathbf{k}}$ conserves mass, momentum and energy as $\mathcal{Q}(f, f)$. However, the H-theorem no longer holds since the truncated approximation $f^{K}$ of a positive function $f$ is generally not positive.

For the VHS collision kernel (2.3) with uncertainty in $b_{\lambda}, Q_{\mathbf{k}}$ can be further expanded as

$$
Q_{\mathbf{k}}(t, \mathbf{x}, \mathbf{v})=\sum_{|\mathbf{i}|,|\mathbf{j}|=0}^{K} S_{\mathbf{k i j}} \int_{\mathbb{R}^{d}} \int_{S^{d-1}}\left|\mathbf{v}-\mathbf{v}_{*}\right|^{\lambda}\left[f_{\mathbf{i}}\left(\mathbf{v}^{\prime}\right) f_{\mathbf{j}}\left(\mathbf{v}_{*}^{\prime}\right)-f_{\mathbf{i}}(\mathbf{v}) f_{\mathbf{j}}\left(\mathbf{v}_{*}\right)\right] \mathrm{d} \sigma \mathrm{d} \mathbf{v}_{*},
$$

with

$$
S_{\mathbf{k i j}}:=\int_{I_{\mathbf{z}}} b_{\lambda}(\mathbf{z}) \Phi_{\mathbf{k}}(\mathbf{z}) \Phi_{\mathbf{i}}(\mathbf{z}) \Phi_{\mathbf{j}}(\mathbf{z}) \pi(\mathbf{z}) \mathrm{d} \mathbf{z}
$$


For the Maxwell boundary condition (2.5) with uncertainty in $T_{w}$ (assume $\mathbf{u}_{w}=0$ for simplicity), $g_{\mathbf{k}}$ is given by

$$
g_{\mathbf{k}}(t, \mathbf{x}, \mathbf{v})=(1-\alpha) f_{\mathbf{k}}(t, \mathbf{x}, \mathbf{v}-2(\mathbf{v} \cdot n) n)+\alpha \sum_{|\mathbf{j}|=0}^{K} D_{\mathbf{k j}}(\mathbf{x}, \mathbf{v}) \int_{\mathbf{v} \cdot n<0} f_{\mathbf{j}}(t, \mathbf{x}, \mathbf{v})|\mathbf{v} \cdot n| \mathrm{d} \mathbf{v}
$$

with

$$
D_{\mathbf{k j}}(\mathbf{x}, \mathbf{v}):=\int_{I_{\mathbf{z}}} \frac{e^{-\frac{\mathbf{v}^{2}}{2 T_{w}(\mathbf{x}, \mathbf{z})}}}{(2 \pi)^{\frac{d-1}{2}} T_{w}^{\frac{d+1}{2}}(\mathbf{x}, \mathbf{z})} \Phi_{\mathbf{k}}(\mathbf{z}) \Phi_{\mathbf{j}}(\mathbf{z}) \pi(\mathbf{z}) \mathrm{d} \mathbf{z} .
$$

In $(3.4)$, since $D_{\mathbf{k j}}$ does not depend on the solution $f_{\mathbf{k}}$, it can be precomputed accurately and stored for repeated use. Likewise, $S_{\mathbf{k i j}}$ in (3.3) can also be precomputed, but even so, the evaluation of $Q_{\mathbf{k}}$ still presents a challenge. A naive, direct computation for each $t$, $\mathbf{x}$, and $\mathbf{k}$ would result in $O\left(N_{K}^{2} N_{\sigma}^{d-1} N_{\mathbf{v}}^{2 d}\right)$ complexity, where $N_{K}=\left(\begin{array}{c}K+n \\ K\end{array}\right)$ is the dimension of $\mathbb{P}_{K}^{n}$ (the set of all $n$-variate polynomials of degree up to $K), N_{\sigma}$ is the number of discrete points in each angular direction, and $N_{\mathbf{v}}$ is the number of points in each velocity dimension. This is, if not impossible, prohibitively expensive.

Remark 3.2. To solve the problem (2.7), an alternative method is to use stochastic collocation [33]: one can apply a deterministic scheme to each sample point in random space, solve them separately to obtain the solution ensemble, and then construct an approximation, e.g., a gPC approximation, to the stochastic solution in the random space. The stochastic Galerkin method, on the other hand, provides better accuracy per stochastic degree of freedom, and with suitably designed fast solvers (see, for instance, the following subsection), is quite competitive with collocation and the costs can be lower for high dimensional random inputs [10].

\subsection{A fast algorithm for the collision operator}

In this subsection, we construct a fast algorithm for evaluating (3.3). We show that the above direct cost $O\left(N_{K}^{2} N_{\sigma}^{d-1} N_{\mathbf{v}}^{2 d}\right)$ can be reduced to $\max \left\{O\left(R_{\mathbf{k}} N_{\sigma}^{d-1} N_{\mathbf{v}}^{d} \log N_{\mathbf{v}}\right), O\left(R_{\mathbf{k}} N_{K} N_{\mathbf{v}}^{d}\right)\right\}$ with $R_{\mathbf{k}} \leq N_{K}$ by leveraging the SVD and the fast spectral method for the deterministic collision operator [28]. This is achieved in two steps.

First, for each fixed $\mathbf{k}$, decompose the symmetric matrix $\left(S_{\mathbf{k i j}}\right)_{N_{K} \times N_{K}}$ as (via a truncated SVD with desired accuracy)

$$
S_{\mathbf{k i j}}=\sum_{r=1}^{R_{\mathbf{k}}} U_{\mathbf{i} r}^{\mathbf{k}} V_{r \mathbf{j}}^{\mathbf{k}}
$$

Substituting it into (3.3) and rearranging terms, we obtain

$$
Q_{\mathbf{k}}(\mathbf{v})=\sum_{r=1}^{R_{\mathbf{k}}} \int_{\mathbb{R}^{d}} \int_{S^{d-1}}\left|\mathbf{v}-\mathbf{v}_{*}\right|^{\lambda}\left[g_{r}^{\mathbf{k}}\left(\mathbf{v}^{\prime}\right) h_{r}^{\mathbf{k}}\left(\mathbf{v}_{*}^{\prime}\right)-g_{r}^{\mathbf{k}}(\mathbf{v}) h_{r}^{\mathbf{k}}\left(\mathbf{v}_{*}\right)\right] \mathrm{d} \sigma \mathrm{d} \mathbf{v}_{*},
$$

with

$$
g_{r}^{\mathbf{k}}(\mathbf{v}):=\sum_{|\mathbf{i}|=0}^{K} U_{\mathbf{i} r}^{\mathbf{k}} f_{\mathbf{i}}(\mathbf{v}), \quad h_{r}^{\mathbf{k}}(\mathbf{v}):=\sum_{|\mathbf{i}|=0}^{K} V_{r \mathbf{i}}^{\mathbf{k}} f_{\mathbf{i}}(\mathbf{v})
$$

Hence we readily reduce the cost from $O\left(N_{K}^{2} N_{\sigma}^{d-1} N_{\mathbf{v}}^{2 d}\right)$ to $\max \left\{O\left(R_{\mathbf{k}} N_{\sigma}^{d-1} N_{\mathbf{v}}^{2 d}\right), O\left(R_{\mathbf{k}} N_{K} N_{\mathbf{v}}^{d}\right)\right\}$, where $R_{\mathbf{k}} \leq N_{K}$ is the numerical rank of matrix $\left(S_{\mathbf{k i j}}\right)_{N_{K} \times N_{K}}$. 
Next, note that (3.5) can be formally written as

$$
Q_{\mathbf{k}}(\mathbf{v})=\sum_{r=1}^{R_{\mathbf{k}}} \mathcal{Q}\left(g_{r}^{\mathbf{k}}, h_{r}^{\mathbf{k}}\right),
$$

and $\mathcal{Q}$ is the deterministic collision operator (2.6) with kernel $B=\left|\mathbf{v}-\mathbf{v}_{*}\right|^{\lambda}$. In [28], a fast Fourier-spectral method in velocity variable $\mathbf{v}$ was developed for (2.2) in the case of 2 D Maxwell molecule $(\lambda=0)$ and 3D hard-sphere molecule $(\lambda=1)$. Applying this method to (3.6) with

slight modification, one can further reduce the cost from $\max \left\{O\left(R_{\mathbf{k}} N_{\sigma}^{d-1} N_{\mathbf{v}}^{2 d}\right), O\left(R_{\mathbf{k}} N_{K} N_{\mathbf{v}}^{d}\right)\right\}$ to $\max \left\{O\left(R_{\mathbf{k}} N_{\sigma}^{d-1} N_{\mathbf{v}}^{d} \log N_{\mathbf{v}}\right), O\left(R_{\mathbf{k}} N_{K} N_{\mathbf{v}}^{d}\right)\right\}$, see appendix for a detailed description (in practice, typically $\left.N_{\sigma} \ll N_{\mathbf{v}}[14,12]\right)$.

Remark 3.3. If the fast Fourier-spectral method is applied directly to compute the integral in (3.3), the total cost of evaluating $Q_{\mathbf{k}}$ would be $O\left(N_{K}^{2} N_{\sigma}^{d-1} N_{\mathbf{v}}^{d} \log N_{\mathbf{v}}\right)$, still more expensive than what we proposed here.

Remark 3.4. The value of $R_{\mathbf{k}}$ is closely related to the properties of $\left\{\Phi_{\mathbf{k}}(\mathbf{z})\right\}$ and $b_{\lambda}(\mathbf{z})$. In our numerical examples using Legendre polynomials, we observe that for prescribed accuracy $10^{-12}$, $R_{\mathbf{k}} \equiv N_{K}$ for $n=1$, and $R_{\mathbf{k}} \leq N_{K}$ for $n=2$ (some $R_{\mathbf{k}}$ can be significantly less than $N_{K}$ and results in further saving). We also mention that the tensor $S_{\mathbf{k i j}}$ itself has some sparse structure owing to the orthogonality of basis and many symmetries, as discussed in [27]. In principle, one can identify its nonzero values and perform computation (3.3) only on those points. The SVD approach we used here is a simple alternative, and is feasible when $N_{K}$ is not very large. Exploring the low-rank and sparsity structure of $S_{\mathbf{k i j}}$, especially for high-dimensional random inputs, will be a subject of future research.

\subsection{Time and spatial discretizations}

A semi-discrete scheme for (3.2) consists of two steps:

- Convection step:

$$
\frac{f_{\mathbf{k}}^{*}-f_{\mathbf{k}}^{n}}{\Delta t}+\mathbf{v} \cdot \nabla_{\mathbf{x}} f_{\mathbf{k}}^{n}=0
$$

- Collision step:

$$
\frac{f_{\mathbf{k}}^{n+1}-f_{\mathbf{k}}^{*}}{\Delta t}=\frac{1}{\mathrm{Kn}} Q_{\mathbf{k}}^{*} .
$$

For the spatial derivative in the convection step, we employ the second-order MUSCL scheme [30]. Second-order accuracy in time can be achieved using Runge-Kutta scheme and Strang splitting.

Remark 3.5. In this paper, we do not consider very small Knudsen number, thus an explicit scheme is used for the collision step. Developing efficient numerical schemes that work uniformly for a wide range of Kn constitutes an active research area called "asymptotic-preserving scheme" [20]. This is currently under consideration in the framework of UQ [19]. 


\subsection{Computation of the moments}

When solving the Boltzmann equation, besides the directly evolved density distribution function, people are also interested in its various moments such as those defined in (2.4). In the stochastic Galerkin method, a remaining issue is: given $f_{\mathbf{k}}$, how to obtain reasonable gPC expansion coefficients for macroscopic quantities? Inspired by [27], we proceed as follows:

- Compute $\rho_{\mathbf{k}}, \mathbf{m}_{\mathbf{k}}$, and $E_{\mathbf{k}}$ by direct integration

$$
\rho_{\mathbf{k}}:=\int_{\mathbb{R}^{d}} f_{\mathbf{k}} \mathrm{d} \mathbf{v}, \quad \mathbf{m}_{\mathbf{k}}:=\int_{\mathbb{R}^{d}} f_{\mathbf{k}} \mathbf{v} \mathrm{d} \mathbf{v}, \quad E_{\mathbf{k}}:=\frac{1}{2} \int_{\mathbb{R}^{d}} f_{\mathbf{k}}|\mathbf{v}|^{2} \mathrm{~d} \mathbf{v} .
$$

- Compute $\rho_{\mathbf{k}}^{-1}$ from

$$
\rho \rho^{-1}=1 \Rightarrow\left(\sum_{|\mathbf{i}|=0}^{K} \rho_{\mathbf{i}} \Phi_{\mathbf{i}}\right)\left(\sum_{|\mathbf{j}|=0}^{K} \rho_{\mathbf{j}}^{-1} \Phi_{\mathbf{j}}\right)=1,
$$

which upon projection amounts to solving the linear system

$$
\sum_{|\mathbf{j}|=0}^{K} a_{\mathbf{k j}} \rho_{\mathbf{j}}^{-1}=\delta_{\mathbf{0 k}}
$$

with

$$
a_{\mathbf{k j}}:=\sum_{|\mathbf{i}|=0}^{K} \rho_{\mathbf{i}} S_{\mathbf{k i j}}^{\prime}, \quad S_{\mathbf{k i j}}^{\prime}:=\int_{I_{\mathbf{z}}} \Phi_{\mathbf{k}}(\mathbf{z}) \Phi_{\mathbf{i}}(\mathbf{z}) \Phi_{\mathbf{j}}(\mathbf{z}) \pi(\mathbf{z}) \mathrm{d} \mathbf{z} .
$$

- Compute $\mathbf{u}_{\mathbf{k}}$ and $T_{\mathbf{k}}$ via

$$
\begin{aligned}
& u_{\mathbf{k}}^{(l)}=\sum_{|\mathbf{i}|, \mathbf{j} \mid=0}^{K} m_{\mathbf{i}}^{(l)} \rho_{\mathbf{j}}^{-1} S_{\mathbf{k i j}}^{\prime}, \\
& T_{\mathbf{k}}=\frac{2}{d} \sum_{|\mathbf{i}|, \mathbf{j} \mid=0}^{K}\left(E_{\mathbf{i}} \rho_{\mathbf{j}}^{-1}-\frac{1}{2} \sum_{l=1}^{d} u_{\mathbf{i}}^{(l)} u_{\mathbf{j}}^{(l)}\right) S_{\mathbf{k i j}}^{\prime},
\end{aligned}
$$

where superscripts $l$ denote the $l$ th component of a $d$-dimensional vector.

Remark 3.6. One can also use the numerical quadrature to approximate the moments. As this step serves as post-processing, we do not elaborate further.

\section{Regularity and numerical accuracy study in the random space}

In this section, we assume $\mathbf{z}=z \in I_{z}$ is one dimensional, and $I_{z}$ has finite support (which is the case, for examples, for the uniform and Beta distributions). We prove that, for the spatially homogeneous equation

$$
\frac{\partial f}{\partial t}=\mathcal{Q}(f, f)
$$

the solution to the Boltzmann equation with uncertainty preserves the regularity in the random space of the initial data. We then establish the spectral convergence of the stochastic Galerkin method. 


\subsection{Regularity in terms of the random variable}

In this subsection we analyze the regularity of the solution in terms of the random variable. The analysis is similar to that in velocity space [13].

Define

$$
\begin{aligned}
& \|f\|_{L_{\mathbf{v}}^{p}}=\left(\int_{\mathbb{R}^{d}}|f|^{p} \mathrm{~d} \mathbf{v}\right)^{\frac{1}{p}}, \quad(1 \leq p<\infty), \quad\|f\|_{L_{z}^{2}}=\left(\int_{I_{z}}|f|^{2} \pi(z) \mathrm{d} z\right)^{\frac{1}{2}}, \\
& \|f\|_{H_{z}^{k}}=\left(\sum_{l=0}^{k}\left\|\partial_{z}^{l} f\right\|_{L_{z}^{2}}^{2}\right)^{\frac{1}{2}}, \quad\|f\|_{k}=\sup _{z \in I_{z}}\left(\sum_{l=0}^{k}\left\|\partial_{z}^{l} f\right\|_{L_{\mathbf{v}}^{2}}^{2}\right)^{\frac{1}{2}} .
\end{aligned}
$$

Our analysis will rely on two basic properties of the collision operator, as described by the following lemma.

Lemma 4.1. Assume that $B$ and $\partial_{z}^{l} B$ (all $z$ derivatives of $B$ ) are $C^{\infty}$ functions in $\mathbf{v}$, bounded in $z$, compactly supported as functions of $\left|\mathbf{v}-\mathbf{v}^{*}\right|$, vanishing for $\left|\mathbf{v}-\mathbf{v}^{*}\right|$ small enough. If $g, h \in L_{\mathbf{v}}^{1} \cap L_{\mathbf{v}}^{2}$, then

$$
\begin{aligned}
& \|\mathcal{Q}(g, h)\|_{L_{\mathbf{v}}^{2}} \leq C_{B}\|g\|_{L_{\mathbf{v}}^{1}}\|h\|_{L_{\mathbf{v}}^{2}}, \quad\|\mathcal{Q}(g, h)\|_{L_{\mathbf{v}}^{2}} \leq C_{B}\|g\|_{L_{\mathbf{v}}^{2}}\|h\|_{L_{\mathbf{v}}^{1}}, \\
& \|\mathcal{Q}(g, h)\|_{L_{\mathbf{v}}^{2}} \leq C_{B}\|g\|_{L_{\mathbf{v}}^{2}}\|h\|_{L_{\mathbf{v}}^{2}}
\end{aligned}
$$

where $C_{B}>0$ only depends on the collision kernel $B$.

The estimate (4.2) was a result of P.L. Lions [23], while (4.3) was a result of BouchutDesvillettes and $\mathrm{Lu}[3,25]$. See also [31]. Since $B$ depends on and is bounded in $z, C_{B}$ is also bounded in $z$.

We state the following theorem.

Theorem 4.2. Assume that $B$ satisfies the assumption in Lemma 4.1, and $\sup _{z \in I_{z}}\left\|f^{0}\right\|_{L_{\mathbf{v}}^{1}} \leq M$, $\left\|\mid f^{0}\right\| \|_{k}<\infty$. Then there exists a constant $C_{k}>0$, only depends on $C_{B}, M$, T, and $\left\|\mid f^{0}\right\| \|_{k}$ such that

$$
\|\mid f\|_{k} \leq C_{k} \quad \text { for any } t \in[0, T] .
$$

Proof. First, due to the conservation property of $\mathcal{Q}$, one has

$$
\|f(t, \cdot, z)\|_{L_{\mathbf{v}}^{1}}=\left\|f^{0}(\cdot, z)\right\|_{L_{\mathbf{v}}^{1}} \leq M
$$

Next, we will use mathematical induction. For $k=0$, multiplying (4.1) by $f$ and integrating on $\mathbf{v}$, and using the Cauchy-Schwartz inequality and (4.2), one obtains

$$
\frac{1}{2} \partial_{t} \int_{\mathbb{R}^{d}} f^{2} \mathrm{~d} \mathbf{v}=\int_{\mathbb{R}^{d}} f \mathcal{Q}(f, f) \mathrm{d} \mathbf{v} \leq\|f\|_{L_{\mathbf{v}}^{2}}\|\mathcal{Q}(f, f)\|_{L_{\mathbf{v}}^{2}} \leq C_{B}\|f\|_{L_{\mathbf{v}}^{1}}\|f\|_{L_{\mathbf{v}}^{2}}^{2} \leq C_{B} M\|f\|_{L_{\mathbf{v}}^{2}}^{2}
$$

Now Gronwall's inequality simply implies that there is a positive constant $C_{0}$ such that (4.4) holds for $k=0$.

Now assume (4.4) holds for all $0 \leq l \leq k$. Define

$$
\mathcal{Q}_{l}(f, g)(\mathbf{v})=\int_{\mathbb{R}^{d}} \int_{S^{d-1}}\left[\partial_{z}^{l} B\left(\mathbf{v}-\mathbf{v}_{*}, \sigma, z\right)\right]\left[f\left(\mathbf{v}^{\prime}\right) g\left(\mathbf{v}_{*}^{\prime}\right)-f(\mathbf{v}) g\left(\mathbf{v}_{*}\right)\right] \mathrm{d} \sigma \mathrm{d} \mathbf{v}_{*},
$$


then $\mathcal{Q}_{l}$ also satisfies the properties listed in Lemma 4.1. We will still use the same constant $C_{B}$ here.

Taking $k+1$-th derivative of $z$ on (4.1) gives

$$
\partial_{t} \partial_{z}^{k+1} f=\sum_{l=0}^{k+1}\left(\begin{array}{c}
k+1 \\
l
\end{array}\right) \sum_{l_{1}=0}^{k+1-l}\left(\begin{array}{c}
k+1-l \\
l_{1}
\end{array}\right) \mathcal{Q}_{l}\left(\partial_{z}^{l_{1}} f, \partial_{z}^{k+1-l-l_{1}} f\right) .
$$

Multiplying (4.8) by $\partial_{z}^{k+1} f$ and integrating over $\mathbf{v}$ yields

$$
\begin{array}{ll} 
& \frac{1}{2} \partial_{t} \int_{\mathbb{R}^{d}}\left(\partial_{z}^{k+1} f\right)^{2} \mathrm{~d} \mathbf{v} \\
\leq & \sum_{l=0}^{k+1}\left(\begin{array}{c}
k+1 \\
l
\end{array}\right) \sum_{l_{1}=0}^{k+1-l}\left(\begin{array}{c}
k+1-l \\
l_{1}
\end{array}\right)\left\|\partial_{z}^{k+1} f\right\|_{L_{\mathbf{v}}^{2}}\left\|\mathcal{Q}_{l}\left(\partial_{z}^{l_{1}} f, \partial_{z}^{k+1-l-l_{1}} f\right)\right\|_{L_{\mathbf{v}}^{2}} \\
\leq \quad & C_{B}\left\|\partial_{z}^{k+1} f\right\|_{L_{\mathbf{v}}^{2}} \sum_{l=0}^{k+1}\left(\begin{array}{c}
k+1 \\
l
\end{array}\right) \sum_{l_{1}=0}^{k+1-l}\left(\begin{array}{c}
k+1-l \\
l_{1}
\end{array}\right)\left\|\partial_{z}^{l_{1}} f\right\|_{L_{\mathbf{v}}^{2}}\left\|\partial_{z}^{k+1-l-l_{1}} f\right\|_{L_{\mathbf{v}}^{2}} \\
= & C_{B}\left\|\partial_{z}^{k+1} f\right\|_{L_{\mathbf{v}}^{2}} \sum_{l=1}^{k+1}\left(\begin{array}{c}
k+1 \\
l
\end{array}\right) \sum_{l_{1}=0}^{k+1-l}\left(\begin{array}{c}
k+1-l \\
l_{1}
\end{array}\right)\left\|\partial_{z}^{l_{1}} f\right\|_{L_{\mathbf{v}}^{2}}\left\|\partial_{z}^{k+1-l-l_{1}} f\right\|_{L_{\mathbf{v}}^{2}} \\
+ & C_{B}\left\|\partial_{z}^{k+1} f\right\|_{L_{\mathbf{v}}^{2}} \sum_{l_{1}=0}^{k+1}\left(\begin{array}{c}
k+1 \\
l_{1}
\end{array}\right)\left\|\partial_{z}^{l_{1}} f\right\|_{L_{\mathbf{v}}^{2}}\left\|\partial_{z}^{k+1-l_{1}} f\right\|_{L_{\mathbf{v}}^{2}} \\
= & C_{B}\left\|\partial_{z}^{k+1} f\right\|_{L_{\mathbf{v}}^{2}} \sum_{l=1}^{k+1}\left(\begin{array}{c}
k+1 \\
l
\end{array}\right) \sum_{l_{1}=0}^{k+1-l}\left(\begin{array}{c}
k+1-l \\
l_{1}
\end{array}\right)\left\|\partial_{z}^{l_{1}} f\right\|_{L_{\mathbf{v}}^{2}}\left\|\partial_{z}^{k+1-l-l_{1}} f\right\|_{L_{\mathbf{v}}^{2}} \\
+\quad & C_{B}\left\|\partial_{z}^{k+1} f\right\|_{L_{\mathbf{v}}^{2}} \sum_{l_{1}=1}^{k}\left(\begin{array}{c}
k+1 \\
l_{1}
\end{array}\right)\left\|\partial_{z}^{l_{1}} f\right\|_{L_{\mathbf{v}}^{2}}\left\|\partial_{z}^{k+1-l_{1}} f\right\|_{L_{\mathbf{v}}^{2}}+2 C_{B}\left\|\partial_{z}^{k+1} f\right\|_{L_{\mathbf{v}}^{2}}^{2}\|f\|_{L_{\mathbf{v}}^{2}} \\
\leq & C_{B} C_{k}^{2}\left\|\partial_{z}^{k+1} f\right\|_{L_{\mathbf{v}}^{2}} \sum_{l=1}^{k+1}\left(\begin{array}{c}
k+1 \\
l
\end{array}\right) \sum_{l_{1}=0}^{k+1-l}\left(\begin{array}{c}
k+1-l \\
l_{1}
\end{array}\right) \\
+ & C_{B} C_{k}^{2}\left\|\partial_{z}^{k+1} f\right\|_{L_{\mathbf{v}}^{2}} \sum_{l_{1}=1}^{k}\left(\begin{array}{c}
k+1 \\
l_{1}
\end{array}\right)+2 C_{B} C_{0}\left\|\partial_{z}^{k+1} f\right\|_{L_{\mathbf{v}}^{2}}^{2} \\
\leq & 2^{2 k} C_{B} C_{k}^{2}\left\|\partial_{z}^{k+1} f\right\|_{L_{\mathbf{v}}^{2}}+2^{k+1} C_{B} C_{k}^{2}\left\|\partial_{z}^{k+1} f\right\|_{L_{\mathbf{v}}^{2}}+2 C_{B} C_{0}\left\|\partial_{z}^{k+1} f\right\|_{L_{\mathbf{v}}^{2}}^{2}
\end{array}
$$

In the first inequality we used the Cauchy-Schwartz inequality. In the second inequality we used (4.3). In the third inequality we used the induction assumption. In the final inequality, we used the identity $\sum_{l=0}^{L}\left(\begin{array}{c}L \\ l\end{array}\right)=(1+1)^{L}=2^{L}$.

Now Gronwall's inequality applies to (4.9) gives (4.4) for $k+1$ with a positive constant $C_{k+1}$. This completes the mathematical induction and consequently proof of the theorem.

Theorem 4.2 shows that the solution to the (spatially homogeneous) Boltzmann equation maintains the regularity in $z$ of the initial data for all time $t>0$.

\subsection{A spectral accuracy analysis}

With $\Phi_{k}$ being an orthonormal basis in $L_{z}^{2}$, one can write

$$
f(t, \mathbf{v}, z)=\sum_{k=0}^{\infty} \hat{f}_{k}(t, \mathbf{v}) \Phi_{k}(z), \quad \text { where } \quad \hat{f}_{k}(t, \mathbf{v})=\int_{I_{z}} f(t, \mathbf{v}, z) \Phi_{k}(z) \pi(z) \mathrm{d} z .
$$


Let $P_{K}$ be the projection operator defined as

$$
P_{K} f(t, \mathbf{v}, z)=\sum_{k=0}^{K} \hat{f}_{k}(t, \mathbf{v}) \Phi_{k}(z) .
$$

We will only consider the case of uniform distribution, so $z \in I_{z}=[-1,1]$ and $\pi(z)=1 / 2$. Accordingly $\Phi_{k}$ are the Legendre polynomials.

Assume $\left\|\left|f^{0} \|\right|_{m+1}\right.$ is bounded, then the Theorem 4.2 implies that $\||f(t, \cdot, \cdot) \||_{m+1}$ is bounded. One has the following estimate on the projection error (see for example $[8,17]$ ):

$$
\sup _{z \in I_{z}}\left|f(t, \mathbf{v}, \cdot)-P_{K} f(t, \mathbf{v}, \cdot)\right| \leq C \frac{\|f(t, \mathbf{v}, \cdot)\|_{H_{z}^{m+1}}}{K^{m}},
$$

where $C$ is independent of $t$ and $\mathbf{v}$. This implies (we suppress the $t$-dependence)

$$
\begin{aligned}
& \int_{I_{z}}\left\|f(\cdot, z)-P_{K} f(\cdot, z)\right\|_{L_{\mathbf{v}}^{2}}^{2} \pi(z) \mathrm{d} z=\int_{I_{z}} \int_{\mathbb{R}^{d}}\left|f(\mathbf{v}, z)-P_{K} f(\mathbf{v}, z)\right|^{2} \mathrm{~d} \mathbf{v} \pi(z) \mathrm{d} z \\
\leq & \frac{C}{K^{2 m}} \int_{I_{z}} \int_{\mathbb{R}^{d}}\|f\|_{H_{z}^{m+1}}^{2} \mathrm{~d} \mathbf{v} \pi(z) \mathrm{d} z=\frac{C}{K^{2 m}} \int_{\mathbb{R}^{d}}\|f\|_{H_{z}^{m+1}}^{2} \mathrm{~d} \mathbf{v} \\
= & \frac{C}{K^{2 m}} \int_{I_{z}} \int_{\mathbb{R}^{d}} \sum_{l=0}^{m+1}\left(\partial_{z}^{l} f\right)^{2} \mathrm{~d} \mathbf{v} \pi(z) \mathrm{d} z \\
\leq & \frac{C C_{k}^{2}}{K^{2 m}} \int_{I_{z}} \pi(z) \mathrm{d} z=\frac{C C_{k}^{2}}{K^{2 m}},=\frac{C}{K^{2 m}}
\end{aligned}
$$

where we used Theorem 4.2 and in the last equality we used a generic constant.

Inserting (4.10) into the Boltzmann equation (4.1) and carrying out the Galerkin projection, one will obtain

$$
\frac{\partial \hat{f}_{k}}{\partial t}=Q_{k}(f, f), \quad k \geq 0
$$

Define the numerical error as

$$
e_{k}=\hat{f}_{k}-f_{k}, \quad 0 \leq k \leq K, \quad \mathbf{e}=\left(e_{0}, e_{1}, \cdots, e_{K}\right)^{T},
$$

then one has the following equation for the error:

$$
\frac{\partial e_{k}}{\partial t}=Q_{k}(f, f)-Q_{k}\left(f^{K}, f^{K}\right) .
$$


We now estimate the right hand side of (4.13) for consistency error.

$$
\begin{aligned}
& \left\|Q_{k}(f, f)-Q_{k}\left(f^{K}, f^{K}\right)\right\|_{L_{\mathbf{v}}^{2}}^{2} \\
= & \int_{\mathbb{R}^{d}}\left(\int_{I_{z}}\left[\mathcal{Q}(f, f)(t, \mathbf{v}, z)-\mathcal{Q}\left(f^{K}, f^{K}\right)(t, \mathbf{v}, z)\right] \Phi_{k}(z) \pi(z) \mathrm{d} z\right)^{2} \mathrm{~d} \mathbf{v} \\
= & \int_{\mathbb{R}^{d}}\left(\int_{I_{z}}\left[\mathcal{Q}\left(f-f^{K}, f\right)(t, \mathbf{v}, z)+\mathcal{Q}\left(f^{K}, f-f^{K}\right)(t, \mathbf{v}, z)\right] \Phi_{k}(z) \pi(z) \mathrm{d} z\right)^{2} \mathrm{~d} \mathbf{v} \\
\leq & \int_{\mathbb{R}^{d}}\left(\int_{I_{z}}\left[\mathcal{Q}\left(f-f^{K}, f\right)(t, \mathbf{v}, z)+\mathcal{Q}\left(f^{K}, f-f^{K}\right)(t, \mathbf{v}, z)\right]^{2} \pi(z) \mathrm{d} z\right)\left(\int_{I_{z}} \Phi_{k}^{2}(z) \pi(z) \mathrm{d} z\right) \mathrm{d} \mathbf{v} \\
= & \int_{I_{z}} \int_{\mathbb{R}^{d}}\left[\mathcal{Q}\left(f-f^{K}, f\right)(t, \mathbf{v}, z)+\mathcal{Q}\left(f^{K}, f-f^{K}\right)(t, \mathbf{v}, z)\right]^{2} \mathrm{~d} \mathbf{v} \pi(z) \mathrm{d} z \\
\leq & 2 \int_{I_{z}}\left(\left\|\mathcal{Q}\left(f-f^{K}, f\right)(t, \cdot, z)\right\|_{L_{\mathbf{v}}^{2}}^{2}+\left\|\mathcal{Q}\left(f^{K}, f-f^{K}\right)(t, \cdot, z)\right\|_{L_{\mathbf{v}}^{2}}^{2}\right) \pi(z) \mathrm{d} z \\
\leq & 2 C_{B}^{2} \int_{I_{z}}\left(\|f\|_{L_{\mathbf{v}}^{2}}^{2}\left\|f-f^{K}\right\|_{L_{\mathbf{v}}^{2}}^{2}+\left\|f^{K}\right\|_{L_{\mathbf{v}}^{2}}^{2}\left\|f-f^{K}\right\|_{L_{\mathbf{v}}^{2}}^{2}\right) \pi(z) \mathrm{d} z \\
\leq & C \int_{I_{z}}\left\|f-f^{K}\right\|_{L_{\mathbf{v}}^{2}}^{2} \pi(z) \mathrm{d} z, \\
\leq & C \int_{I_{z}}\left(\left\|f-P_{K} f\right\|_{L_{\mathbf{v}}^{2}}^{2}+\left\|P_{K} f-f^{K}\right\|_{L_{\mathbf{v}}^{2}}^{2}\right) \pi(z) \mathrm{d} z \\
\leq & C\left\{\frac{1}{K^{2 m}}+\|\mathbf{e}\|_{L_{\mathbf{v}}^{2}}^{2}\right\} .
\end{aligned}
$$

In the above estimation, we used Cauchy-Schwartz inequality in the first inequality, and Lemma 4.1 in the third inequality. In the fourth inequality we used the boundedness of $f$ proved in the previous subsection and the uniform boundedness assumption of $f^{K}$ in $K$ (this is an assumption that we cannot prove), and in the last inequality we used the projection error (4.12).

Now multiplying both sides of (4.13) by $e_{k}$, summing over $k$, and using Cauchy-Schwartz inequality on the right hand side, one can easily derive the following inequality:

$$
\frac{\partial}{\partial t}\|\mathbf{e}\|_{L_{\mathbf{v}}^{2}} \leq C\left\{\frac{1}{K^{m}}+\|\mathbf{e}\|_{L_{\mathbf{v}}^{2}}\right\} .
$$

By Gronwall's inequality one obtains

$$
\|\mathbf{e}(t)\|_{L_{\mathbf{v}}^{2}} \leq e^{C t}\left\{\frac{1}{K^{m}}+\|\mathbf{e}(0)\|_{L_{\mathbf{v}}^{2}}\right\} .
$$

Finally,

$$
\left\|f-f^{K}\right\|_{L_{\mathbf{v}}^{2}} \leq\left\|f-P_{K} f\right\|_{L_{\mathbf{v}}^{2}}+\left\|P_{K} f-f^{K}\right\|_{L_{\mathbf{v}}^{2}} .
$$

This, together with (4.14) gives

$$
\left\|f-f^{K}\right\|_{L_{\mathbf{v}}^{2}} \leq C(t)\left\{\frac{1}{K^{m}}+\|\mathbf{e}(0)\|_{L_{\mathbf{v}}^{2}}\right\},
$$

which shows the spectral accuracy for smooth solutions.

Remark 4.3. Clearly for spectral accuracy, one needs $\int\|\mathbf{e}(0)\|_{L_{\mathbf{v}}^{2}} d z \leq C / K^{m}$. In practice, one chooses $f_{k}(0, \mathbf{v})=\hat{f}_{k}(0, \mathbf{v})$, for all $k=0, \cdots, K$, then $\mathbf{e}(0)=0$.

Remark 4.4. One can combine the spectral accuracy analysis in the random space and the spectral accuracy analysis for the collision operator established in [28] to obtain a spectral accuracy for the combined random space and velocity space. We omit the straightforward details. 


\section{$5 \quad$ Numerical examples}

In this section, we present several numerical examples to illustrate the validity of the proposed scheme. We will consider both spatially homogeneous and inhomogeneous problems, wherein the randomness can come from collision kernel, initial data, or boundary data. For simplicity, we will always assume the random variable $\mathbf{z}$ obeys the uniform distribution on $[-1,1]^{n}$ with $n$ up to 2, thus the Legendre polynomials are used as gPC basis. Given the gPC coefficients $f_{\mathbf{k}}$ of $f$, the statistical information mean, variance, and standard deviation are retrieved as

$$
\mathbb{E}[f] \approx f_{\mathbf{0}}, \quad \operatorname{Var}[f] \approx \sum_{|\mathbf{k}|=1}^{K} f_{\mathbf{k}}^{2}, \quad S[f] \approx \sqrt{\sum_{|\mathbf{k}|=1}^{K} f_{\mathbf{k}}^{2}} .
$$

\subsection{The spatially homogeneous BGK equation}

Example 1. We first consider the homogeneous BGK equation

with random collision kernel

$$
\frac{\partial f}{\partial t}=B(\mathbf{z})(\mathcal{M}-f), \quad v \in \mathbb{R}
$$

$$
B(\mathbf{z})=1+s_{1} z_{1}+s_{2} z_{2}, \quad s_{1}=0.2, s_{2}=0.1,
$$

and deterministic initial condition

$$
f^{0}(v)=v^{2} e^{-v^{2}}
$$

This is a particularly simple example where the Maxwellian $\mathcal{M}$ neither depends on z nor changes in time. Figure 1 shows the mean and standard deviation of $f$ obtained using gPC up to 7 th degree. The fourth-order Runge-Kutta scheme is applied for time discretization. The velocity space is discretized using 64 points.
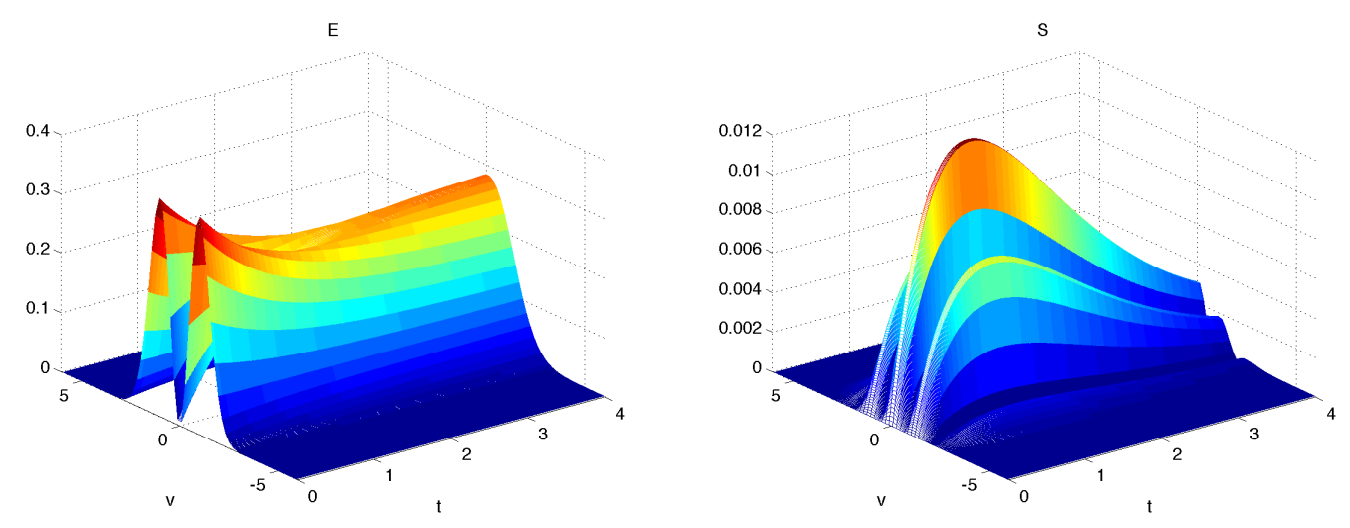

Figure 1: Example 1. Left: $\mathbb{E}[f](t, v)$. Right: $S[f](t, v) . K=7, N_{v}=64, \Delta t=0.2 / 32$, RK-4 for time discretization.

To further verify the accuracy of the scheme, we conduct the convergence analysis by comparing with the exact solution. Results are gathered in Figure 2, where we clearly observe the spectral accuracy in random space and desired accuracy in time. 

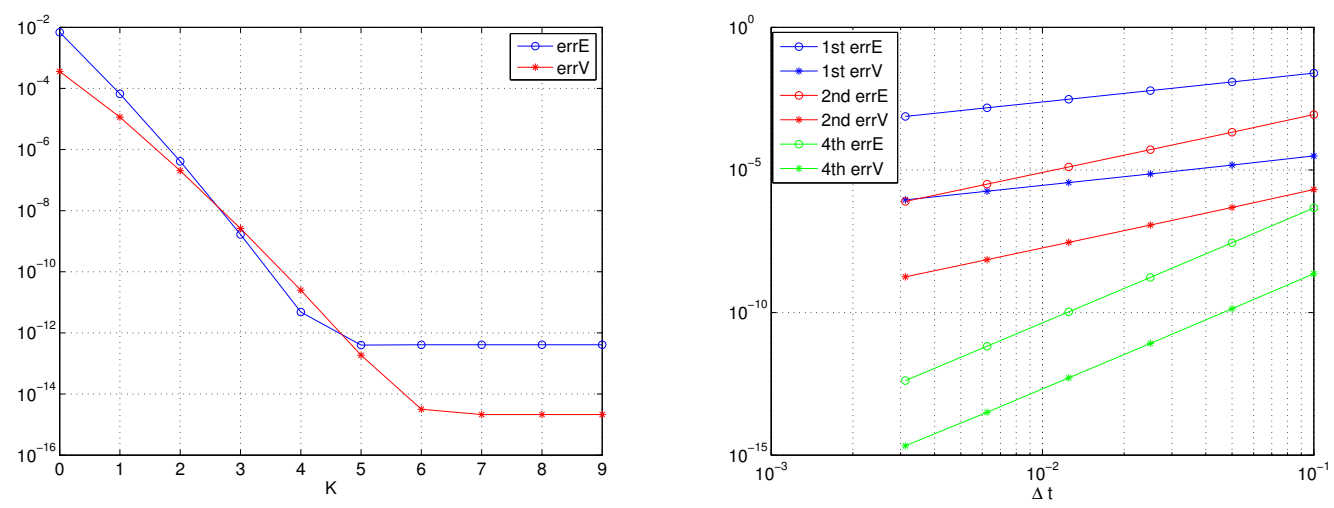

Figure 2: Example 1. Left: spectral accuracy in $K(\Delta t=0.2 / 64, \mathrm{RK}-4$ for time discretization). Right: 1st, 2nd, and 4th order accuracy in time $(K=9)$. errE $=\left\|\mathbb{E}[f]-\mathbb{E}\left[f^{\text {ext }}\right]\right\|_{l^{1}(t, v)}$, $\operatorname{errV}=\left\|\operatorname{Var}[f]-\operatorname{Var}\left[f^{\text {ext }}\right]\right\|_{l^{1}(t, v)}$.

\subsection{The spatially homogeneous Boltzmann equation}

We now consider the homogeneous Boltzmann equation. In this and the rest of examples, we always assume 2D Maxwell molecules, i.e., $\mathbf{v} \in \mathbb{R}^{2}$ and $\lambda=0$ in (2.3). Extension to 3D is straightforward.

\subsubsection{Random collision kernel}

Example 2. Assume the collision kernel

$$
B(z)=1+s z, \quad s=0.2
$$

and the initial data

$$
f^{0}(\mathbf{v})=\frac{1}{2 \pi^{2}} \mathbf{v}^{2} e^{-\mathbf{v}^{2}}
$$

Figure 3 shows the mean and standard deviation of $f$ at $t=2.5$ obtained using gPC up to 7th degree. The second-order Heun's method is applied for time discretization. The velocity space is discretized on $64 \times 64$ mesh, and $N_{\sigma}=8$ for angular discretization.

Without uncertainty, this is a famous benchmark example for the Boltzmann equation where the exact solution can be constructed [11]. However, it is not the case with a random collision kernel. We therefore compare our solution with the stochastic collocation [33] using 20 GaussLegendre quadrature points. The solutions at different time are plotted in Figure 4.

\subsubsection{Random initial data}

Example 3. Assume constant kernel $B=1$ but random initial data

$$
f^{0}(\mathbf{v}, z)=\frac{1}{2 \pi^{2}} \mathbf{v}^{2} e^{-\frac{\mathbf{v}^{2}}{1+s z}}, \quad s=0.2,
$$

we perform the same test as above. The results are shown in Figures 5 and 6 . We see that the mean of the solution is more or less the same no matter whether the uncertainty is in collision 

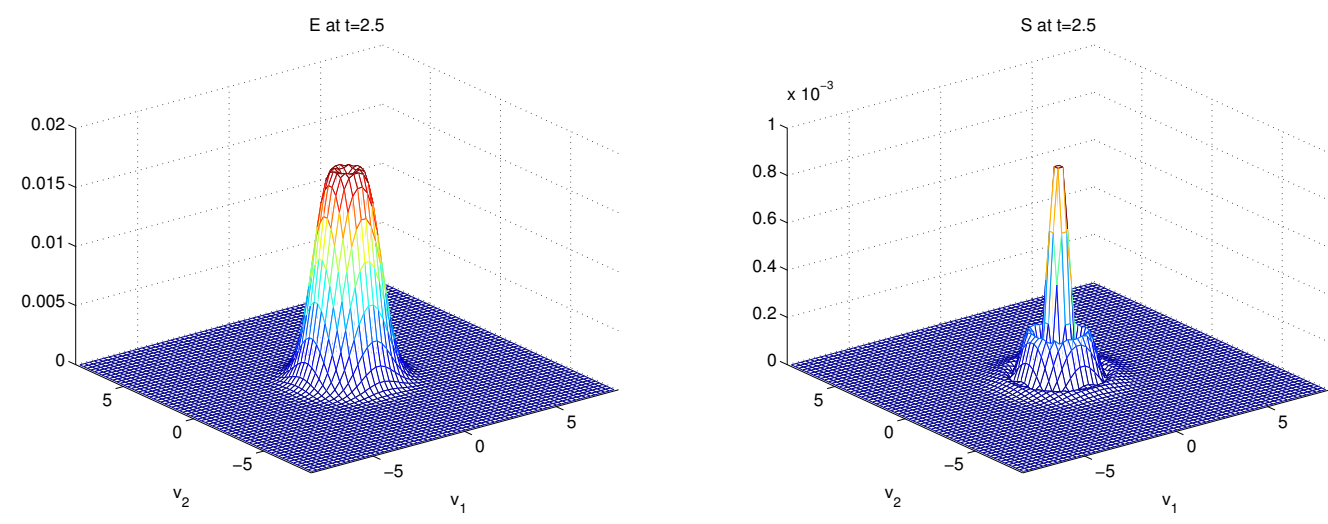

Figure 3: Example 2. Left: $\mathbb{E}[f](t=2.5, \mathbf{v})$. Right: $S[f](t=2.5, \mathbf{v}) . K=7, N_{\mathbf{v}}=64, N_{\sigma}=8$, $\Delta t=0.1$, Heun for time discretization.
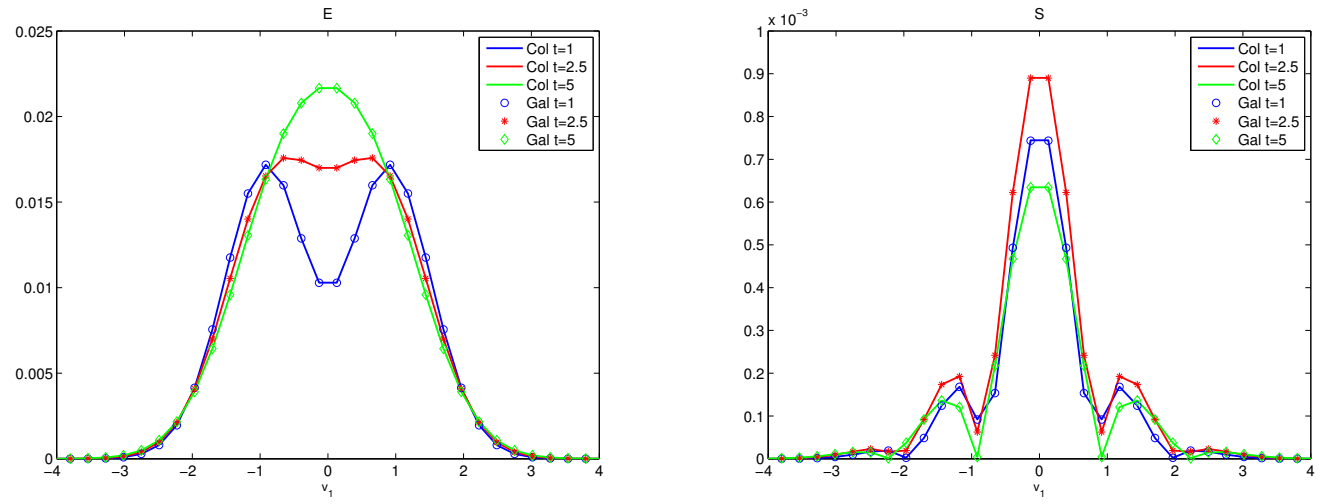

Figure 4: Example 2. $\mathbb{E}[f]$ and $S[f]$ at $v_{2}=0$. "Col" stands for collocation, "Gal" stands for Galerkin. Heun is used for Galerkin, RK-4 is used for collocation. 
kernel or initial data, whereas the standard deviation exhibits quite different behavior in the two cases.
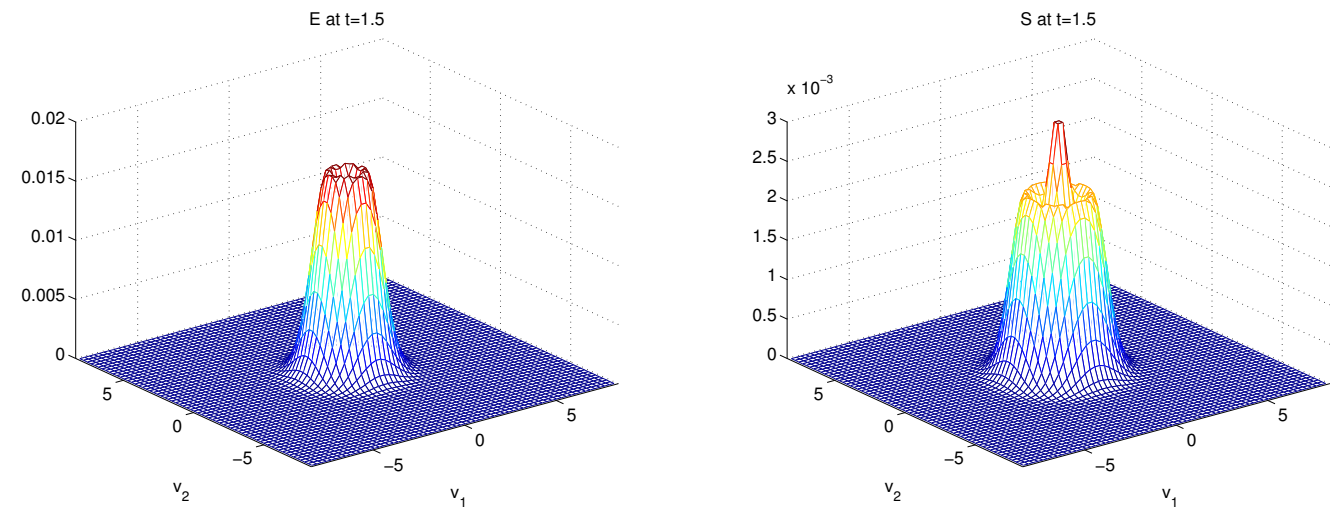

Figure 5: Example 3. Left: $\mathbb{E}[f](t=1.5, \mathbf{v})$. Right: $S[f](t=1.5, \mathbf{v}) . K=7, N_{\mathbf{v}}=64, N_{\sigma}=8$, $\Delta t=0.1$, Heun for time discretization.
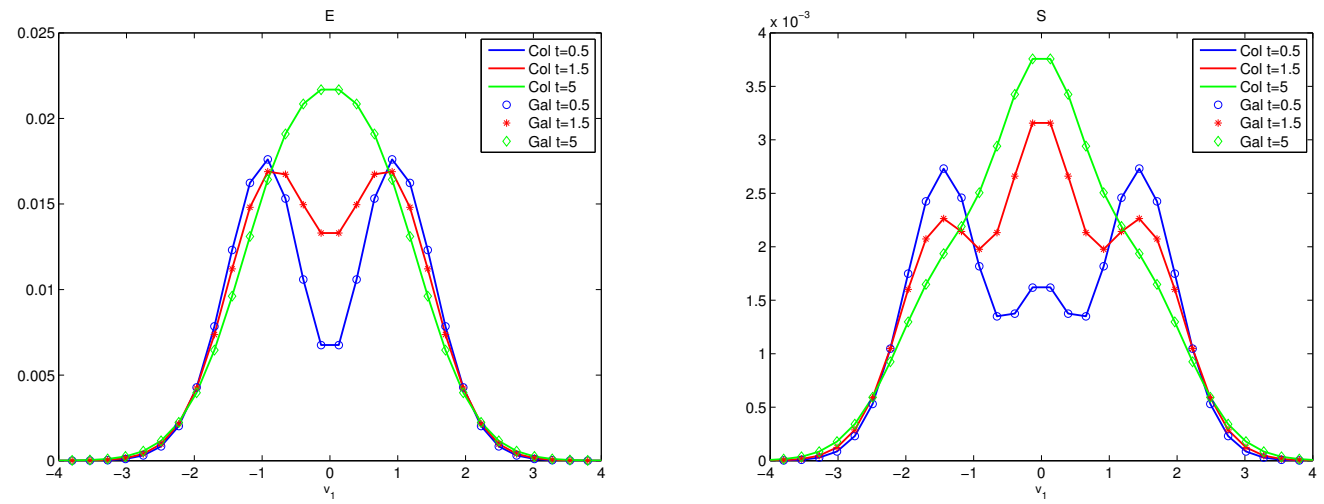

Figure 6: Example 3. $\mathbb{E}[f]$ and $S[f]$ at $v_{2}=0$. "Col" stands for collocation, "Gal" stands for Galerkin. Heun is used for Galerkin, RK-4 is used for collocation.

\subsection{The spatially inhomogeneous Boltzmann equation}

We finally consider the full Boltzmann equation and assume 1D in $\mathbf{x}$. For not too small Knudsen number $\mathrm{Kn}$, time step $\Delta t$ is mainly controlled by the CFL condition from the convection part. In the following examples, we take a fixed CFL number 0.8 and implement the MUSCL scheme with minmod slope limiter for spatial discretization.

\subsubsection{Random collision kernel}

Example 4. Assume the collision kernel

$$
B(z)=1+s z, \quad s=0.6,
$$


the continuous initial data

$$
f^{0}(x, \mathbf{v})=\frac{\rho^{0}(x)}{4 \pi T^{0}(x)}\left(e^{-\frac{\left|\mathbf{v}-\mathbf{u}^{0}(x)\right|^{2}}{2 T^{0}(x)}}+e^{-\frac{\left|\mathbf{v}+\mathbf{u}^{0}(x)\right|^{2}}{2 T^{0}(x)}}\right), \quad x \in[0,1],
$$

where

$$
\rho^{0}(x)=\frac{2+\sin (2 \pi x)}{3}, \quad \mathbf{u}^{0}=(0.2,0), \quad T^{0}=\frac{3+\cos (2 \pi x)}{4},
$$

periodic boundary condition in $x$, and $\mathrm{Kn}=0.1$. We compare the solution obtained by stochastic Galerkin with that of stochastic collocation on a finer mesh using 20 Gauss-Legendre quadrature points. The macroscopic quantities density, bulk velocity and temperature are depicted in Figure 7, where we observe good agreement between two methods.
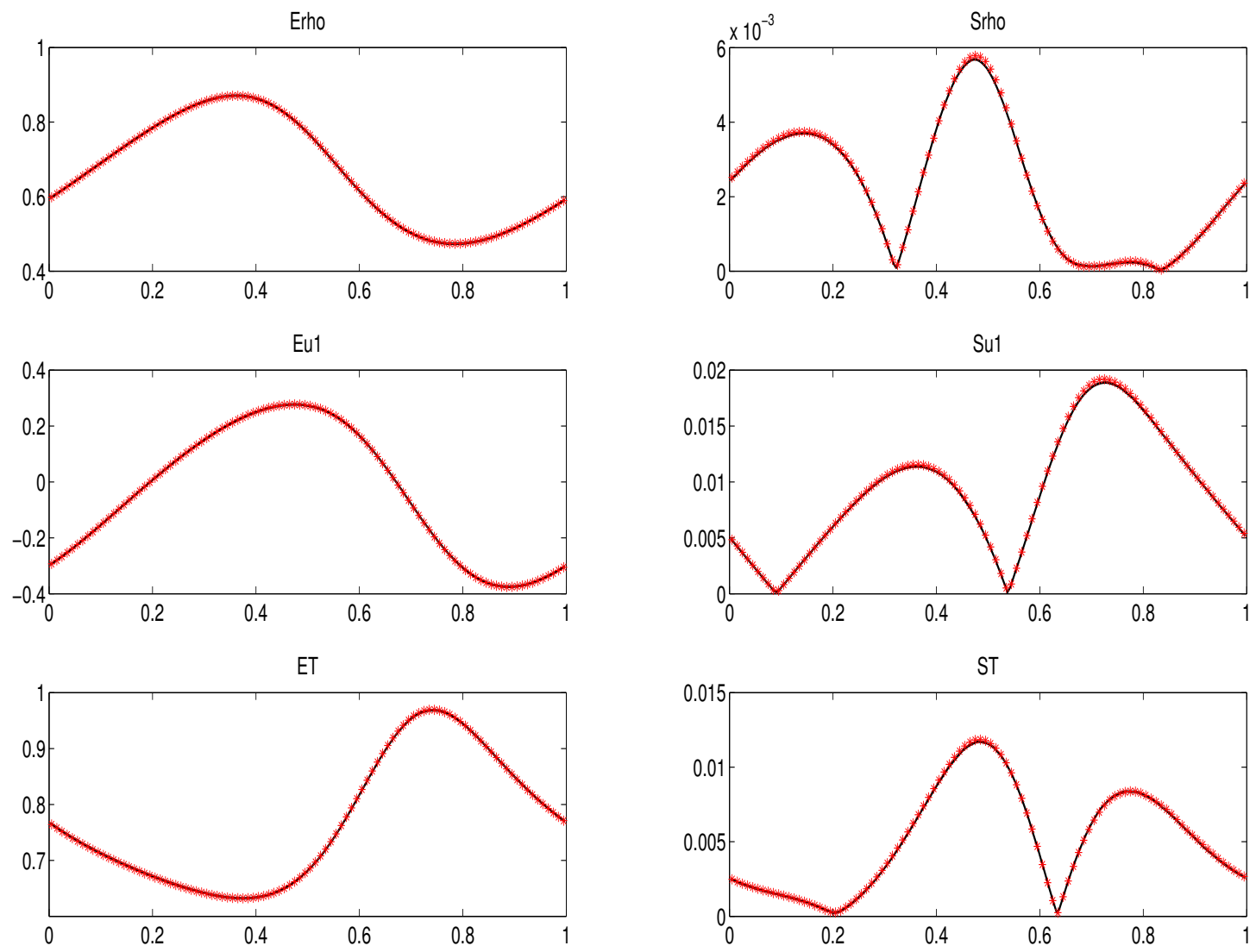

Figure 7: Example 4. Solutions at $t=0.2$. Left column: mean of density, bulk velocity (first component), and temperature. Right column: standard deviation of density, bulk velocity (first component), and temperature. Solid line: collocation with $N_{z}=20, N_{\mathbf{v}}=64, N_{\sigma}=8$, $N_{x}=200$. Red star: Galerkin with $K=7, N_{\mathbf{v}}=32, N_{\sigma}=4, N_{x}=100$.

\subsubsection{Random initial data}

In this subsection, we assume the randomness is only in initial data. 
Example 5 (Shock tube problem).

Consider the equilibrium initial condition with random macroscopic quantities:

$$
\begin{cases}\rho_{l}=1+s_{1}\left(\frac{z+1}{2}\right), & u_{l}=0, \quad T_{l}=1+s_{2} z, \quad x \leq 0.5 \\ \rho_{r}=0.125, \quad u_{r}=0, & T_{r}=0.25, \quad x>0.5\end{cases}
$$

To illustrate the effects of uncertainty in different macroscopic quantities, we examine two cases (1) $s_{1}=0.2, s_{2}=0 ;(2) s_{1}=0.2, s_{2}=0.1$. The results are shown, respectively, in Figures 8 and 9. The wiggles in the solution are due to the fact that a global gPC is used to approximate a discontinuous function, and can be improved by using local adaptive basis such as [32, 26].
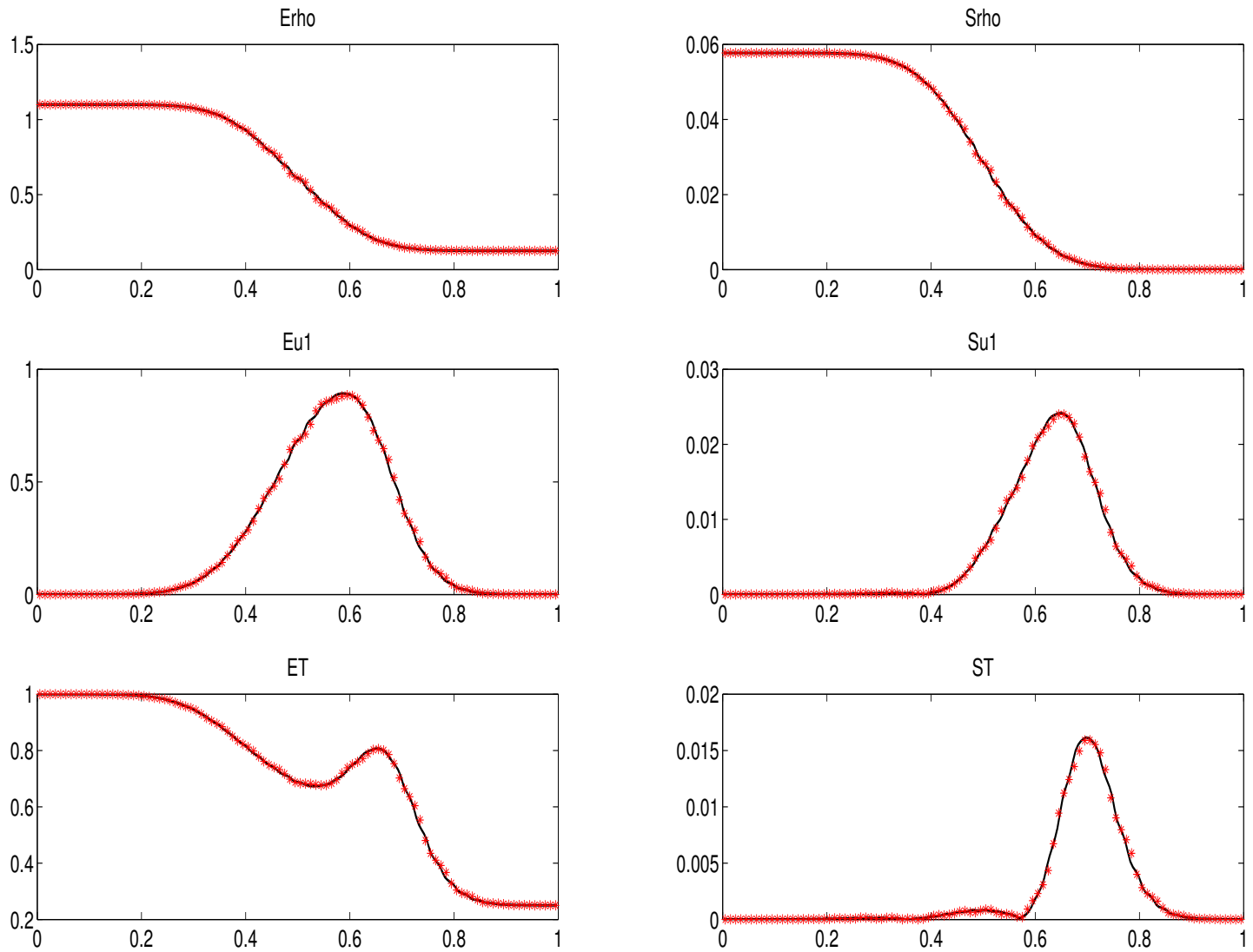

Figure 8: Example 5: case (1) $s_{1}=0.2, s_{2}=0$. Solutions at $t=0.1$. Left column: mean of density, bulk velocity (first component), and temperature. Right column: standard deviation of density, bulk velocity (first component), and temperature. Solid line: collocation with $N_{z}=20$, $N_{\mathbf{v}}=64, N_{\sigma}=8, N_{x}=200$. Red star: Galerkin with $K=7, N_{\mathbf{v}}=32, N_{\sigma}=4, N_{x}=100$. 

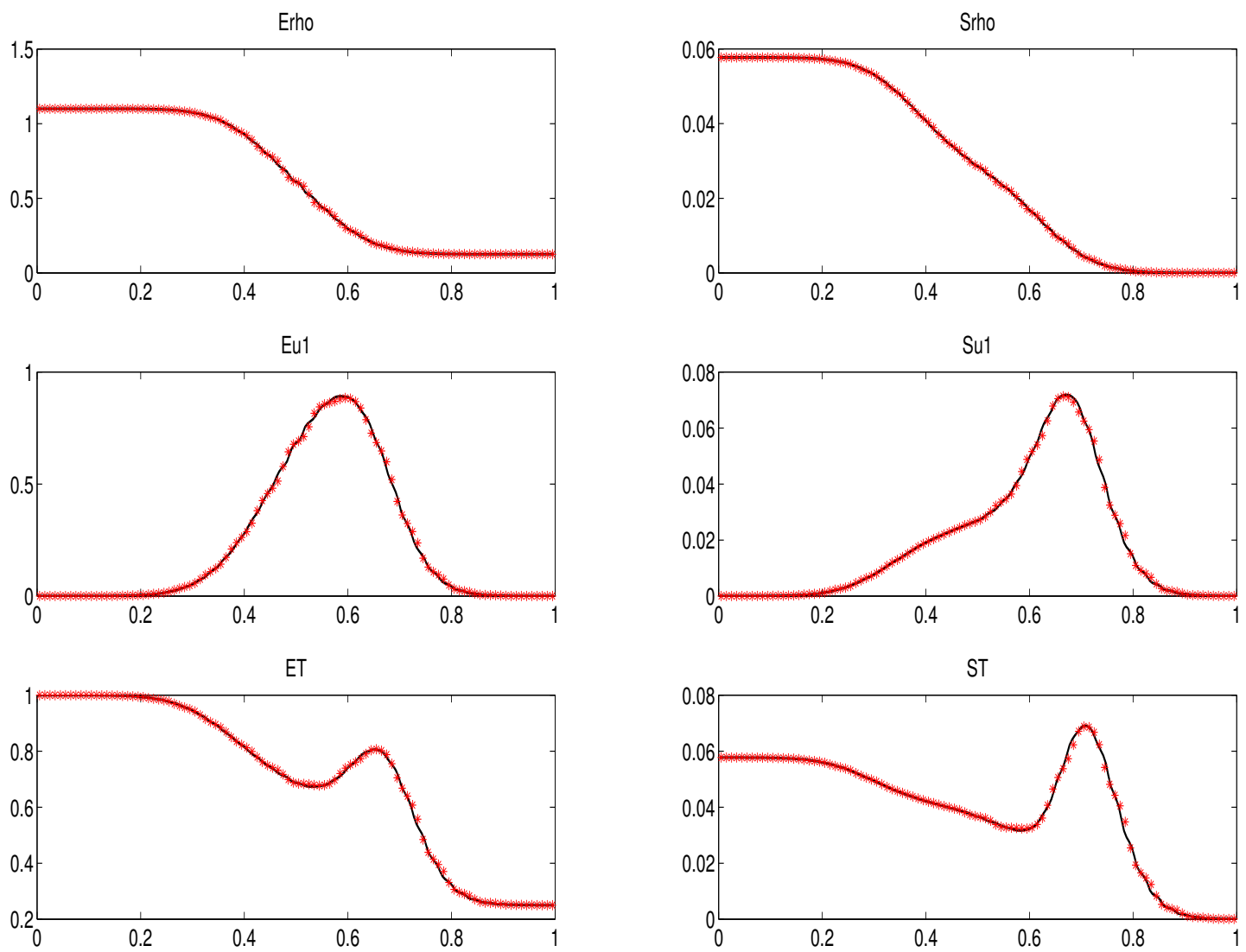

Figure 9: Example 5: case (2) $s_{1}=0.2, s_{2}=0.1$. Solutions at $t=0.1$. Left column: mean of density, bulk velocity (first component), and temperature. Right column: standard deviation of density, bulk velocity (first component), and temperature. Solid line: collocation with $N_{z}=20$, $N_{\mathbf{v}}=64, N_{\sigma}=8, N_{x}=200$. Red star: Galerkin with $K=7, N_{\mathbf{v}}=32, N_{\sigma}=4, N_{x}=100$. 
Example 6. Consider the same initial data as in (5.1), (5.2) except

$$
\begin{aligned}
& \rho^{0}(x, \mathbf{z})=\frac{2+\sin (2 \pi x)+\frac{1}{2} \sin (4 \pi x) z_{1}+\frac{1}{3} \sin (6 \pi x) z_{2}}{3}, \\
& T^{0}(x, \mathbf{z})=\frac{3+\cos (2 \pi x)+\frac{1}{2} \cos (4 \pi x) z_{1}+\frac{1}{3} \cos (6 \pi x) z_{2}}{4} .
\end{aligned}
$$

These are chosen to mimic the K-L expansion. Periodic boundary condition is assumed in $x$. In the stochastic collocation, 10 Gauss-Legendre quadrature points are used for each random dimension. See results in Figure 10.
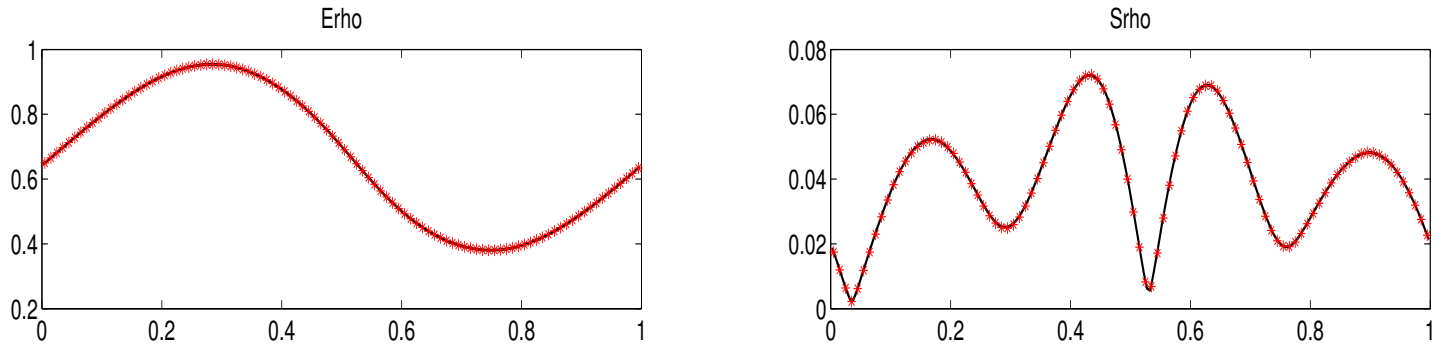

Eu1
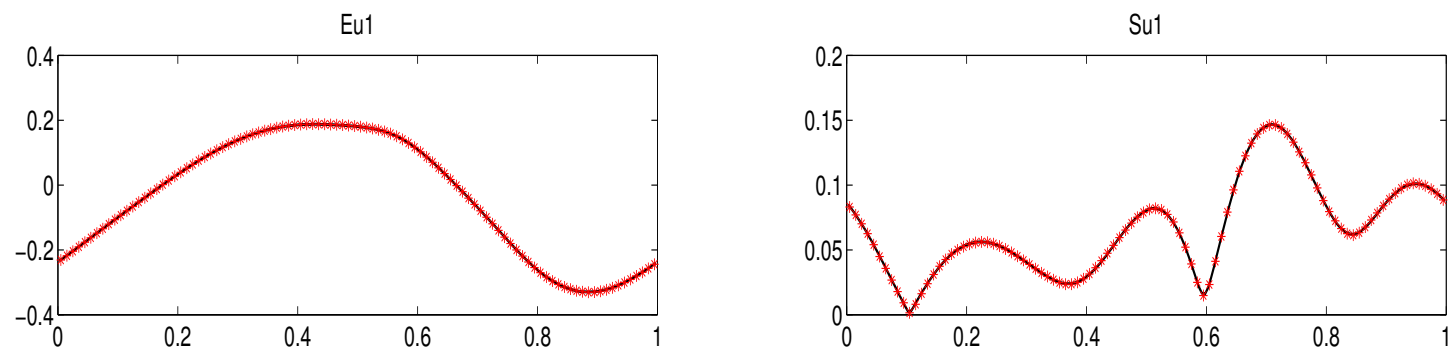

ET
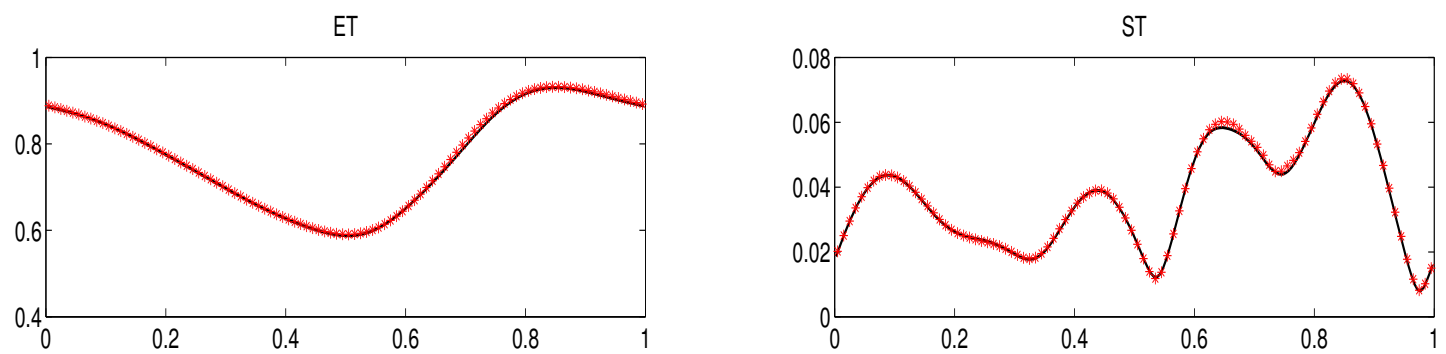

Figure 10: Example 6. Solutions at $t=0.1$. Left column: mean of density, bulk velocity (first component), and temperature. Right column: standard deviation of density, bulk velocity (first component), and temperature. Solid line: collocation with $N_{\mathbf{z}}=10, N_{\mathbf{v}}=64, N_{\sigma}=8$, $N_{x}=200$. Red star: Galerkin with $K=5, N_{\mathbf{v}}=32, N_{\sigma}=4, N_{x}=100$.

\subsubsection{Random boundary data}

Example 7 (Sudden heating problem).

The gas is initially in a constant state

$$
f^{0}(x, \mathbf{v})=\frac{1}{2 \pi T^{0}} e^{-\frac{\mathbf{v}^{2}}{2 T^{0}}}, \quad T^{0}=1, \quad x \in[0,1] .
$$


At time $t=0$, suddenly change the wall temperature at left boundary to

$$
T_{w}(z)=2\left(T_{0}+s z\right), \quad s=0.2 .
$$

Assume purely diffusive Maxwell boundary condition at $x=0$, and $\mathrm{Kn}=0.1$.

The deterministic version of this problem has been considered by many authors $[1,15,12]$, where they all observed that with the sudden rise of wall temperature, the gas close to the wall is heated and accordingly the pressure there rises sharply, which pushes the gas away from the wall and a shock wave propagates into the domain. The mean of our solution also exhibits the similar behavior (see Figure 11). Meanwhile, the standard deviation of the solution allows us to predict the propagation of uncertainties quantitatively.
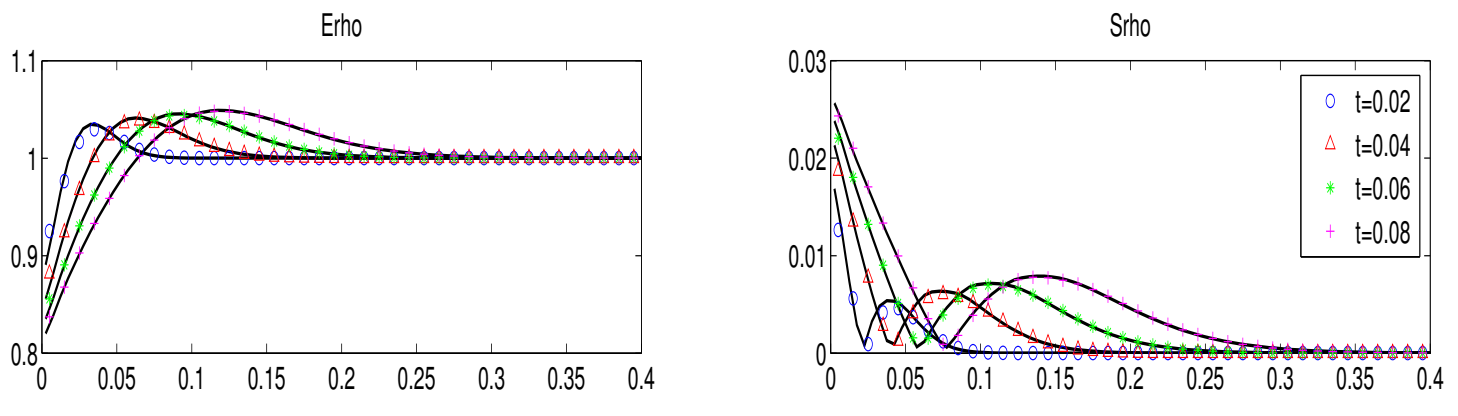

Eu1
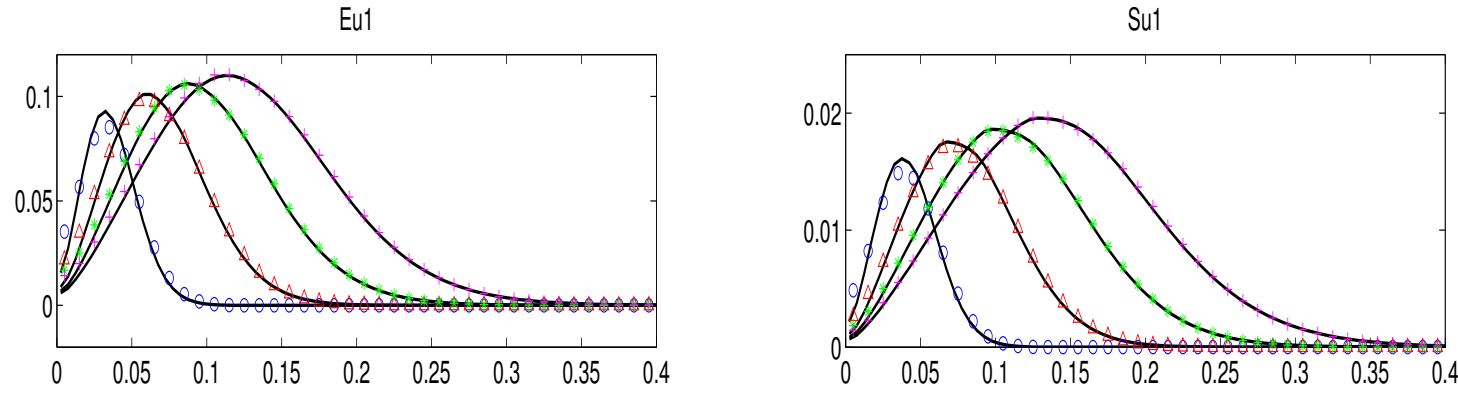

ET
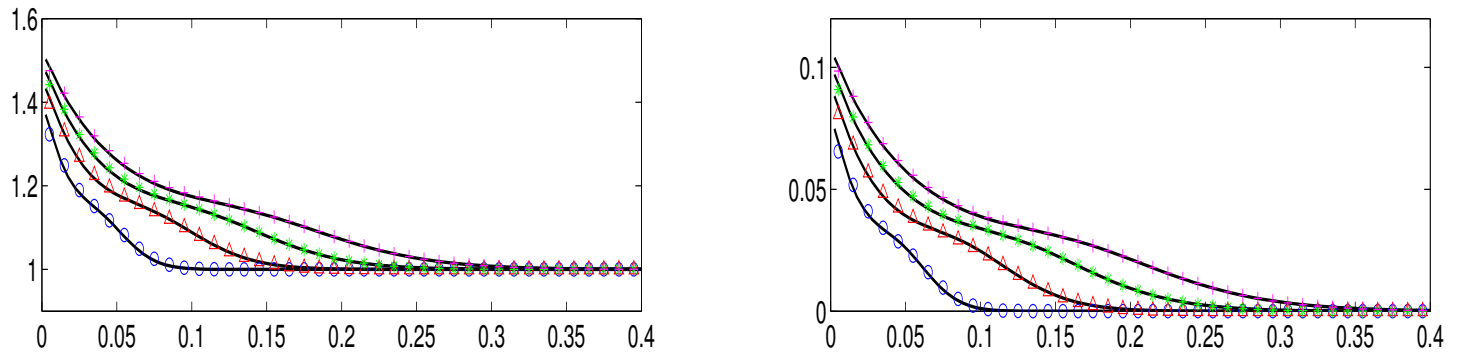

Figure 11: Example 7. Left column: mean of density, bulk velocity (first component), and temperature. Right column: standard deviation of density, bulk velocity (first component), and temperature. Solid line: collocation with $N_{z}=20, N_{\mathbf{v}}=64, N_{\sigma}=8, N_{x}=200$. Other legends are the Galerkin solutions at different time with $K=7, N_{\mathbf{v}}=32, N_{\sigma}=4, N_{x}=100$. 


\section{Conclusion}

We have introduced a gPC based stochastic Galerkin method for the nonlinear Boltzmann equation with random inputs. For the first time, we are able to quantify the uncertainties arising from collision kernel, initial data, or boundary data. Along the way, we proposed a fast algorithm to accelerate the computation of the collision operator under the Galerkin projection. In the spatially homogeneous case, we also established the regularity and proved the spectral accuracy in the random space of the stochastic Galerkin method. This paper serves as an initial attempt to conduct UQ for nonlinear kinetic equations. Many interesting problems are open or currently under investigation, such as dimension reduction for high dimensional random inputs, asymptotic-preserving schemes that are efficient near the fluid dynamic regime, etc.

\section{Appendix: fast Fourier-spectral method for the collision operator $\mathcal{Q}(g, h)$}

In this appendix, we outline the fast Fourier-spectral method for computing the collision operator (3.6). First it suffices to consider an operator of the form

$$
\mathcal{Q}(g, h)(\mathbf{v})=\int_{\mathbb{R}^{d}} \int_{S^{d-1}}\left|\mathbf{v}-\mathbf{v}_{*}\right|^{\lambda}\left[g\left(\mathbf{v}^{\prime}\right) h\left(\mathbf{v}_{*}^{\prime}\right)-g(\mathbf{v}) h\left(\mathbf{v}_{*}\right)\right] \mathrm{d} \sigma \mathrm{d} \mathbf{v}_{*},
$$

where $g$ and $h$ are arbitrary functions of $\mathbf{v}$ (not necessarily positive). The starting point in [28] is to transform the above integral into a Carleman-like representation:

$$
\mathcal{Q}(g, h)(\mathbf{v})=\int_{\mathbb{R}^{d}} \int_{\mathbb{R}^{d}} \tilde{B}(\mathbf{x}, \mathbf{y}) \delta(\mathbf{x} \cdot \mathbf{y})\left[g\left(\mathbf{v}^{\prime}\right) h\left(\mathbf{v}_{*}^{\prime}\right)-g(\mathbf{v}) h\left(\mathbf{v}_{*}\right)\right] \mathrm{d} \mathbf{x} \mathrm{d} \mathbf{y},
$$

where $\mathbf{v}_{*}=\mathbf{v}+\mathbf{x}+\mathbf{y}, \mathbf{v}^{\prime}=\mathbf{v}+\mathbf{x}, \mathbf{v}_{*}^{\prime}=\mathbf{v}+\mathbf{y}$, and $\tilde{B}(\mathbf{x}, \mathbf{y})=2^{d-1}|\mathbf{x}+\mathbf{y}|^{\lambda+2-d}$, so for 2D Maxwell molecule $(d=2, \lambda=0)$ and $3 \mathrm{D}$ hard sphere molecule $(d=3, \lambda=1)$, one has $\tilde{B} \equiv C$. The construction of Fourier spectral method for (7.1) can then be summarized as follows (assume $\tilde{B}=1)$ :

- Truncate the integral to a ball $\mathcal{B}_{R}$ (for both $\mathbf{x}$ and $\mathbf{y}$ ) with $R \geq 2 S, \mathcal{B}_{S} \approx \operatorname{supp}_{\mathbf{v}}(g, h)$.

- Periodize $g, h$ on the domain $\mathcal{D}_{L}=[-L, L]^{d}$. Choose $L \geq \frac{3 \sqrt{2}+1}{2} S$ for anti-aliasing [29].

- Approximate $g, h$ respectively by truncated Fourier series (note here $k$ is a multi-dimensional index and is different from the main text):

$$
g(\mathbf{v})=\sum_{k} \hat{g}_{k} e^{i \frac{\pi}{L} k \cdot \mathbf{v}}, \quad h(\mathbf{v})=\sum_{k} \hat{h}_{k} e^{i \frac{\pi}{L} k \cdot \mathbf{v}}, \quad k=-\frac{N_{\mathbf{v}}}{2}, \ldots, \frac{N_{\mathbf{v}}}{2}-1 .
$$

- Substitute (7.2) into (7.1), and perform the standard Galerkin projection. The $k$-th mode of the Fourier expansion of $\mathcal{Q}$ is given by

$$
\hat{\mathcal{Q}}_{k}=\sum_{l+m=k}[\beta(l, m)-\beta(m, m)] \hat{g}_{l} \hat{h}_{m}, \quad l, m, k=-\frac{N_{\mathbf{v}}}{2}, \ldots, \frac{N_{\mathbf{v}}}{2}-1,
$$

with the kernel mode

$$
\beta(l, m)=\int_{\mathcal{B}_{R}} \int_{\mathcal{B}_{R}} \delta(\mathbf{x} \cdot \mathbf{y}) e^{i \frac{\pi}{L} l \cdot \mathbf{x}} e^{i \frac{\pi}{L} m \cdot \mathbf{y}} \mathrm{d} \mathbf{x} \mathrm{d} \mathbf{y} .
$$


It is clear that a direct computation of (7.3) (for all $k$ ) would require $O\left(N_{\mathbf{v}}^{2 d}\right)$ complexity. But if we can find a low-rank separated expansion of $\beta$ as

$$
\beta(l, m) \approx \sum_{r=1}^{M} A_{r}(l) B_{r}(m),
$$

then the weighted convolution in (7.3) can be rendered into a pure convolution. Hence the cost will be reduced to $O\left(M N_{\mathbf{v}}^{d} \log N_{\mathbf{v}}\right)$ by using the Fast Fourier Transform.

Now the question left is: how to find a decomposition as in (7.5)? We will explain the idea for 2D Maxwell molecule (readers are referred to [28] for other cases). In (7.4), expanding $\mathbf{x}, \mathbf{y}$ in polar coordinates, we have

$$
\beta(l, m)=\frac{1}{4} \int_{S^{1}} \int_{S^{1}} \delta\left(\sigma_{1} \cdot \sigma_{2}\right) \phi\left(l \cdot \sigma_{1}\right) \phi\left(m \cdot \sigma_{2}\right) \mathrm{d} \sigma_{1} \mathrm{~d} \sigma_{2}=\int_{0}^{\pi} \phi\left(l \cdot \sigma_{\theta}\right) \phi\left(\sqrt{|m|^{2}-\left(m \cdot \sigma_{\theta}\right)^{2}}\right) \mathrm{d} \theta,
$$

where

$$
\phi(s):=\int_{-R}^{R} e^{i \frac{\pi}{L} \rho s} \mathrm{~d} \rho=2 R \operatorname{Sinc}\left(\frac{\pi}{L} R s\right), \quad \sigma_{\theta}:=(\cos \theta, \sin \theta) .
$$

Note that the right hand side of (7.6) is a single integral in $\theta$ and the integrand is readily in decoupled form (also $\pi$-periodic in $\theta$ ). Therefore, we can approximate $\beta$ by a uniform quadrature rule:

$$
\beta(l, m) \approx\left(\frac{\pi}{N_{\sigma}}\right) \sum_{r=1}^{N_{\sigma}} \phi\left(l \cdot \sigma_{\theta_{r}}\right) \phi\left(\sqrt{|m|^{2}-\left(m \cdot \sigma_{\theta_{r}}\right)^{2}}\right),
$$

hence resulting in the desired form.

\section{References}

[1] K. Aoki, Y. Sone, K. Nishino, and H. Sugimoto. Numerical analysis of unsteady motion of a rarefied gas caused by sudden changes of wall temperature with special interest in the propagation of a discontinuity in the velocity distribution function. In A. E. Beylich, editor, Rarefied Gas Dynamics, pages 222-231, 1991.

[2] G. A. Bird. Molecular Gas Dynamics and the Direct Simulation of Gas Flows. Clarendon Press, Oxford, 1994.

[3] F. Bouchut and L. Desvillettes. A proof of the smoothing properties of the positive part of Boltzmann's kernel. Rev. Mat. Iberoamericana, 14:47-61, 1998.

[4] C. Cercignani. The Boltzmann Equation and Its Applications. Springer-Verlag, New York, 1988.

[5] C. Cercignani. Rarefied Gas Dynamics: From Basic Concepts to Actual Calculations. Cambridge University Press, Cambridge, 2000.

[6] C. Cercignani, R. Illner, and M. Pulvirenti. The Mathematical Theory of Dilute Gases. Springer-Verlag, 1994.

[7] S. Chapman and T. G. Cowling. The Mathematical Theory of Non-Uniform Gases. Cambridge University Press, Cambridge, third edition, 1991. 
[8] B. Despres, G. Poette, and D. Lucor. Robust uncertainty propagation in systems of conservation laws with the entropy closure method. In Uncertainty quantification in computational fluid dynamics, volume 92 of Lect. Notes Comput. Sci. Eng., pages 105-149. Springer, Heidelberg, 2013.

[9] G. Dimarco and L. Pareschi. Numerical methods for kinetic equations. Acta Numer., 23:369-520, 2014.

[10] H. Elman, C. Miller, E. Phipps, and R. Tuminaro. Assessment of collocation and Galerkin approaches to linear diffusion equations with random data. Int. J. Uncertainty Quantification, 1:19-33, 2011.

[11] M. H. Ernst. Exact solutions of the nonlinear Boltzmann equation. J. Stat. Phys., 34:10011017, 1984.

[12] F. Filbet. On deterministic approximation of the Boltzmann equation in a bounded domain. Multiscale Model. Simul., 10:792-817, 2012.

[13] F. Filbet and C. Mouhot. Analysis of spectral methods for the homogeneous Boltzmann equation. Trans. Amer. Math. Soc., 363(4):1947-1980, 2011.

[14] F. Filbet, C. Mouhot, and L. Pareschi. Solving the Boltzmann equation in NlogN. SIAM J. Sci. Comput., 28:1029-1053, 2006.

[15] I. Gamba and S. H. Tharkabhushanam. Shock and boundary structure formation by spectral-Lagrangian methods for the inhomogeneous Boltzmann transport equation. $J$. Comput. Math., 28:430-460, 2010.

[16] R. G. Ghanem and P. D. Spanos. Stochastic Finite Elements: A Spectral Approach. SpringerVerlag, New York, 1991.

[17] J. Hesthaven and R. Kirby. Filtering in Legendre spectral methods. Math. Comp., 77:1425$1452,2008$.

[18] J. Hirschfelder, R. Bird, and E. Spotz. The transport properties for non-polar gases. J. Chem. Phys., 16:968-981, 1948.

[19] J. Hu, S. Jin, and R. Shu. Asymptotic-preserving stochastic Galerkin schemes for the Boltzmann equation with uncertainty. preprint, 2015.

[20] S. Jin. Asymptotic preserving (AP) schemes for multiscale kinetic and hyperbolic equations: a review. Riv. Mat. Univ. Parma, 3:177-216, 2012.

[21] S. Jin, D. Xiu, and X. Zhu. Asymptotic-preserving methods for hyperbolic and transport equations with random inputs and diffusive scalings. J. Comput. Phys., 289:35-52, 2015.

[22] K. Koura and H. Matsumoto. Variable soft sphere molecular model for inverse-power-law or Lennard-Jones potential. Phys. Fluids A, 3:2459-2465, 1991.

[23] P.-L. Lions. Compactness in Boltzmann's equation via Fourier integral operators and applications. I, II. J. Math. Kyoto Univ., 34(2):391-427, 429-461, 1994. 
[24] M. Loève. Probability Theory. Springer-Verlag, New York, fourth edition, 1977.

[25] X. Lu. A direct method for the regularity of the gain term in the Boltzmann equation. $J$. Math. Anal. Appl., 228:409-435, 1998.

[26] O. Le Maitre, O. Knio, H. Najm, and R. Ghanem. Uncertainty propagation using WienerHaar expansions. J. Comput. Phys., 197:28-57, 2004.

[27] O. P. Le Maitre and O. M. Knio. Spectral Methods for Uncertainty Quantification: With Applications to Computational Fluid Dynamics. Springer, 2010.

[28] C. Mouhot and L. Pareschi. Fast algorithms for computing the Boltzmann collision operator. Math. Comp., 75:1833-1852, 2006.

[29] L. Pareschi and G. Russo. Numerical solution of the Boltzmann equation I: spectrally accurate approximation of the collision operator. SIAM J. Numer. Anal., 37:1217-1245, 2000.

[30] B. van Leer. Towards the ultimate conservative difference scheme V. A second order sequel to Godunov's method. J. Comput. Phys., 32:101-136, 1979.

[31] C. Villani. A review of mathematical topics in collisional kinetic theory. In S. Friedlander and D. Serre, editors, Handbook of Mathematical Fluid Mechanics, volume I, pages 71-305. North-Holland, 2002.

[32] X. Wan and G. E. Karniadakis. An adaptive multi-element generalized polynomial chaos method for stochastic differential equations. J. Comput. Phys., 209:617-642, 2005.

[33] D. Xiu. Numerical Methods for Stochastic Computations. Princeton University Press, New Jersey, 2010. 